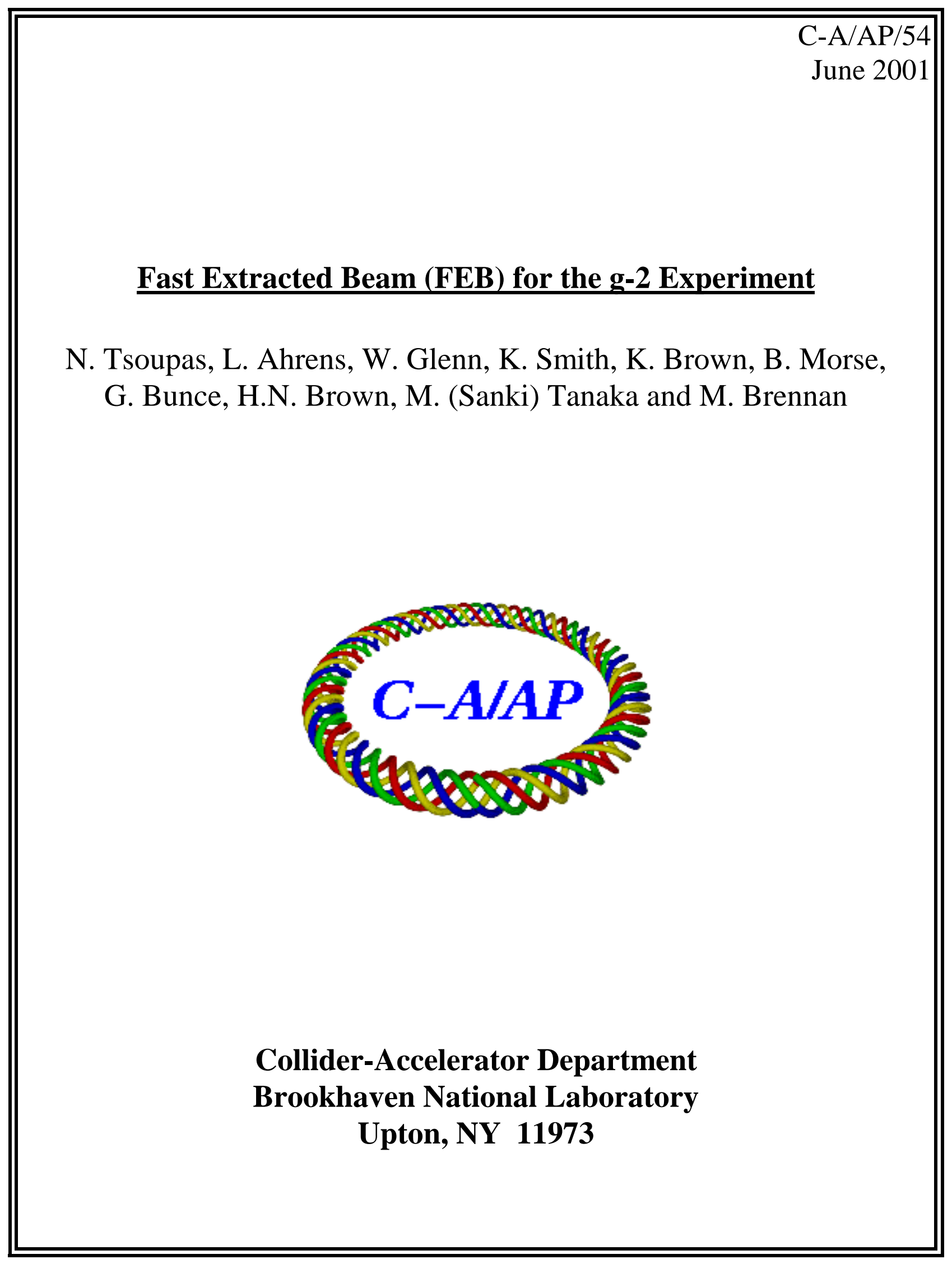




\title{
FAST EXTRACTED BEAM (FEB) FOR THE g-2 EXPERIMENT
}

\author{
N. Tsoupas, L. Ahrens, W. Glenn, K. Smith, K. Brown B. Morse
}

G. Bunce, H.N.Brown, M. (Sanki) Tanaka, and M. Brennan

\begin{abstract}
Proton bunches were extracted from the AGS synchrotron to bombard the V-target[1] for $\pi+$ production to be used in the g-2 experiment[2]. Each of the proton bunches was $\sim 80 \mathrm{nsec}$ long, contained $\sim 6 \times 10^{12}$ protons, with a momentum $\mathrm{p}=24.02 \mathrm{GeV} / \mathrm{c}$ and was transported to the Vtarget via the U-V beam transport line[3] .

This tech-note describes the acceleration process of the proton bunches, and the fast extraction mechanism of the beam bunches from the AGS synchrotron, during the the g-2 experiment which was performed in the time period, December1999 to March 2000.
\end{abstract}

\section{Acceleration stages of the proton beam}

The proton beam underwent the following acceleration stages to reach the final momentum of $24.02 \mathrm{GeV} / \mathrm{c}$.

1. Proton beam in the form of $\mathrm{H}^{-1}$ ions, was extracted from a magnetron ion source [4] at an energy of $35 \mathrm{keV}$ and then was injected into a Radio Frequency Quadrupole (RFQ).

2. The RFQ [5] accelerated the $\mathrm{H}^{-1}$ beam to an energy of $750 \mathrm{keV}$. The accelerated beam was "properly chopped" with the fast chopper [6] located after the RFQ, before it was injected into the $200 \mathrm{MeV}, 200 \mathrm{MHz}$ Linear accelerator (LINAC) [7] (see item \#3 below).

The function of the fast chopper is to turn the beam "on and off" so that the "unchopped" section of the beam, when injected into the AGS-Booster, (see item \#4 below) falls exactly into the circulating bucket of the AGS-Booster. The chopped $\mathrm{H}^{-1}$ beam is lost but at lower energy $(750 \mathrm{keV})$ and in a controlled way.

3. The LINAC accelerated the proton beam to $200 \mathrm{MeV}$, and generated a beam pulse (macro-pulse) $\sim 500 \mu \mathrm{sec}$ long with an average beam current of $35 \mathrm{~mA}$.

The macro-pulse has a fine structure which consists of bunches $1 \mathrm{nsec}$ wide (micropulses) and spaced by $5 \mathrm{nsec}(200 \mathrm{MHz})$.

The macro-pulse, was injected to fill the single bucket of the AGS-Booster synchrotron [8] which acts as a pre-accelerator of the AGS synchrotron. Just before the $\mathrm{H}^{-1}$ beam is injected into the AGS-Booster, it is stripped of its two electrons into a proton, by a carbon foil which is located in the proton injection section of the AGS-Booster and intercepting a " bumped closed orbit".

It takes few hundred turns of the bucket in the AGS-Booster to be filled by the "chopped beam" emerging from the LINAC.

1 This average beam current corresponds to a beam which is "not chopped" by the fast chopper, prior to its injection into the LINAC (see item \#2 above.) 
4. The AGS-Booster synchrotron which operated with two Radio Frequency (RF) cavities ("Band III") at harmonic $(\mathrm{h}=1)$ accelerated the single proton bunch (which was injected from the LINAC), to a momentum of $2.72 \mathrm{GeV} / \mathrm{c}$.

In order to reduce the peak current of the beam, a second RF system ("Band II") operates at harmonic $(\mathrm{h}=2)$ and phased $180^{\circ}$ relative to the ("Band III") system. This second RF system ("Band II") eliminates high charge densities which may appear within a bucket and may cause beam instabilities.

This single bunch was subsequently injected into the AGS which was operating with a single RF cavity at a harmonic $(\mathrm{h}=6)$.

The steps 1 to 4 were repeated six times (six Booster cycles 2 ), until six buckets of the AGS were filled. During the "filling process" the AGS main magnets were operating at "injection energy". At the end of each Booster Acceleration cycle, and approximately $10 \mathrm{msec}$ prior to Booster extraction, the Booster-AGS "synchro" and "cogging" circuit locks the Booster RF to AGS RF ("synchro") and aligns the single Booster RF bucket with the proper AGS receiving bucket.

5. During beam injection into the AGS the AGS RF system operated with two RF stations (out of 10 total) at harmonic $(h=6)$ to receive each of the six single-bunchtransfers from the Booster.

After the injection of the six bunches from the Booster into the AGS, each of the six circulating bunches was split into two, thus generating twelve circulating beam bunches. The bunch splitting, from six bunches into twelve, is necessary because of limitations of peak beam-current at the $\mathrm{V}$-target and is desired by the experiment to reduce instantaneous intensity.

This bunch splitting is performed while the AGS is still on the injection porch, by introducing the remaining eight $\mathrm{RF}$ cavities which operate at harmonic $(\mathrm{h}=12)$ and phased by $180^{\circ}$ relative to the two RF cavities which operate at harmonic $(h=6)$. The gap voltage of these eight stations is raised adiabatically while, simultaneously, the gap voltage of the two cavities running at a harmonic $(h=6)$, is reduced adiabatically. This operation essentially cleaves each $h=6$ bunch into two $h=12$ bunches.

Figure 1 shows the time evolution of the longitudinal charge density, as measured by the "Wall Current Monitor", of each of the six bunches circulating in the AGS, from the time that the first bunch is injected into the AGS to the time the last bunch is extracted from the AGS.

Each horizontal trace shown in figure 1 corresponds to a time interval of two consecutive orbits of the bunches in the AGS. Two consecutive traces in figure 1 differ by $\sim 15 \mathrm{msec}$, thus this "mountain-plot" shows the time evolution of the bunches at time intervals of $15 \mathrm{msec}$. The vertical time span starts from the time the first of the six bunches is injected into the AGS to the time the last of the twelve bunches is extracted from the AGS.

From the values of the vertical axis one can see that the last bunch is injected into the AGS $800 \mathrm{msec}$ after the injection of the first bunch. Notice also the bunch splitting, from 6 into 12, occurs simultaneously for all the bunches.

\footnotetext{
${ }^{2} \mathrm{~A}$ Booster cycle is the time between two consecutive injections from the LINAC and it is approximately $\sim 150$ msec
} 
Following the bunch splitting all 12 bunches were accelerated to the final momentum $24.02 \mathrm{GeV} / \mathrm{c}$ with all $8 \mathrm{RF}$ cavities still running at the harmonic $(\mathrm{h}=12)$, and subsequently, each of the 12 bunches is extracted sequentially. Note in figure 1 the disappearance of each mountain (in the vertical direction) corresponds to the extraction of a bunch from the AGS.

The steps 1 to 5 above describe the acceleration stages of the proton beam. The next section describes briefly the AGS magnet-cycle and the optical properties of the AGS during the acceleration.

Then follows a description of the fast extraction process of the 12 bunches from the AGS to the V-target of the $\mathrm{g}-2$ experiment.

\section{AGS-Magnet-Cycle, Optics and RF control during Beam Acceleration}

The magnet cycle used during the acceleration is shown in figure 2 which plots the Bfield of the AGS main magnets as a function of time.

The beam injection from the Booster to AGS occurs in the early part of the magnet cycle (0 to $\sim 800$ msec see Fig. 2) which is called "front porch or dwell field".

The bunch splitting, from 6 bunches to 12 mentioned earlier, takes place also in the last section of the "front porch".

The rising part of the B vs time curve (Fig. 2) which lasts $\sim 400 \mathrm{msec}$ (from $\sim 950 \mathrm{msec}$ to $\sim 1350$ msec) corresponds to the acceleration of the beam, and the flat section "Flat-Top" of this curve (from $\sim 1350$ to $1850 \mathrm{msec}$ ), corresponds to the coasting of the beam at the maximum energy where the beam extraction takes place.

The optical functions of the AGS (horizontal and vertical tunes $\mathrm{Q}_{\mathrm{x}, \mathrm{y}}$ and chromaticities $\xi_{\mathrm{x}, \mathrm{y}}$ ) which are set by the user, during the AGS cycle are shown in figures. 3 and 4.

These values of the optical parameters as shown in Figs. 3 and 4 are the settings requested by the user and may not coincide with the measured values of the tunes and chromaticities.

Accurate values of the tunes and chromaticities can be obtained by measurements and be compared with theoretical data. An example of such measurements and comparison with theoretical predictions appears in [9].

The optical functions $\mathrm{Q}_{\mathrm{x}, \mathrm{y}}$ and $\xi_{\mathrm{x}, \mathrm{y}}$ as shown in Figs. 3 and 4, have been optimized by the user to maximize the beam current in the AGS, and minimize the beam losses throughout the magnet cycle.

Mechanical constraints on the $\mathrm{V}$-target set a maximum limit on the current that is allowed to be delivered on the V-target. This maximum limit on the beam current, is set to $7.5 \times 10^{12}$ protons/bunch for a total of $60 \times 10^{12}$ protons/(per AGS cycle).

The normalized beam emittance at $95 \%$ beam intensity, was measured to be $(40 \pm 5) \pi . \mathrm{mm} . \mathrm{mrad}$ for both the horizontal and vertical planes. The emittance measurements were obtained from measured beam profiles [10] in the AGS synchrotron.

The RF system, which controls the RF cavities, provides the mechanism for the bunch splitting mentioned earlier, as well as the acceleration and stability of the beam during the AGS main-magnet-cycle.

The gap-volts of the RF cavities, the counter-phasing, and the radial steering function which controls the average radius of the circulating beam in the AGS, during the main-magnet-cycle are shown in Figures 5, 6, and 7 respectively. 
For the "g-2 operations", the gap volts function controls the gap voltage of the $h=12$ RF cavities, and the counter phasing function controls the voltage $\mathrm{b}^{3}$ of the $\mathrm{h}=6 \mathrm{RF}$ cavities.

In addition to the $(\mathrm{h}=6)$ and $(\mathrm{h}=12) \mathrm{RF}$ stations a VHF (Very High Frequency) cavity[20] is also used to provide controlled longitudinal emittance blow-up on the injection porch and after transition.

On the injection porch, longitudinal injection mismatch will cause dipole and quadrupole oscillations to occur. In the course of filamenting the bunch phase space, hot spots can develop in the bunch. The resulting tune spread due to space charge may be too large to accommodate, thus leading to beam loss. The operation of the VHF cavity acts to continually smooth and control the evolution of the emittance blow-up. The resulting bunch evolves in a smoother fashion than it would otherwise, and thus space charge induced tune spread is controlled and minimized. The longitudinal charge distribution of a single bunch is measured by the "Wall Current Monitor" and can be displayed on an oscilloscope or a monitor. Such an evolution of the charge distribution of the bunch, (while the VHF cavity is acting upon the bunch), as a function of the revolution number (vertical axis), is shown by the various traces in figure 8 .

The maximum emittance blow-up which can be tolerated is related to the maximum momentum spread which can be accommodated at the AGS transition where the "gamma-jump" system introduces a large dispersion wave into the lattice. Eventually the resultant transverse beam size at transition sets an upper limit to the dynamic aperture of the AGS.

Following transition, the VHF system may also be used to provide controlled longitudinal emittance blow-up to deal with aspects of beam control. As at injection, longitudinal mismatch following transition and the resulting filamentation would lead to a less than "smooth" bunch. While space charge tune shift is not a concern at high momentum beam ( $24 \mathrm{GeV} / \mathrm{c})$ the VHF system can nonetheless act to control and smooth the filamentation process ultimately producing smoother bunches than might otherwise appear. "Smooth" variations of the beam intensity in a bunch is also desired by the g-2 experiment.

There has also been evidence of a coupled bunch instability occurring after transition. As the threshold for this beam instability is related to bunch length, using the VHF system to again provide a smooth controlled blow-up of the bunch emittance ${ }^{4}$, can cause the coupled bunch instability to extinguish more quickly than it otherwise would.

\section{Fast Extracted Beam (FEB) from the AGS Synchrotron}

Just after the completion of the beam acceleration, and while all 12 bunches circulate in the AGS with a momentum of $24.02 \mathrm{GeV} / \mathrm{c}$, the bunches may be extracted from the AGS synchrotron with the Fast Extracted Beam (FEB) process.

This section describes:

a) The design of the fast beam extraction

b) The time sequence and the function of the various events that occur during the fast beam extraction, and

c) The beam optics of the U-V beam transport line.

\section{A) Design of FEB [11,12] and devices used.}

3 The start triggers for these two functions are different $\{$ the gap volts is triggered after a delay from the (AGS Pre Pulse) APP, and the counter phasing after a delay from $\left.\mathrm{T}_{0}\right\}$ therefore their time scales cannot be compared directly. 4 There are future plans to measure the beam emittance during the AGS cycle. 
The design of the FEB is based on the following operations which are applied on the circulating beam bunches:

a) Generate the $\mathrm{G}$ and $\mathrm{H}$ beam-orbit bumps:

While the beam bunches circulate in the AGS, their center of charge describe an orbit which is referred to as "closed orbit" or "equilibrium orbit". Just before "beamextraction, 5 ( $~ 3.5 \mathrm{msec})$ the equilibrium orbit is displaced ("locally bumped"), at two sections of the AGS ring, thus forming a new equilibrium orbit which is distorted. The first section of the distorted equilibrium orbit is formed around the SS-G10 straight section of the AGS and is referred to as "G09-bump", and the other section is formed around the SS-H10 straight section and is referred to as "H11-bump". An experimental measurement of a distorted equilibrium orbit is shown in figure 9. The vertical axis of this graph represents the average radial displacement, from the "ideal orbit", of the center of charge of all the circulating bunches, and the horizontal axis represents the azimuthal location of the Beam Position Monitors (BPM's) which are the devices that measure this radial orbit displacement.

Note the beam displacement around the G10 and H10 straight sections, when both the G09-bump and H11-bump are powered to generate this particular beam displacement. The G09-bump displaces the beam orbit by $\sim 60 \mathrm{~mm}$ from the "ideal orbit" and on the outside of the AGS ring, and allows the bunches to pass through the aperture of the G10 kicker [11] (see below) which is positioned along the Straight Section-G10. The H11-bump displaces the orbit by $\sim 48 \mathrm{~mm}$, on the outside, from the ideal orbit thus bringing the bunches close to the septum of the H10 magnet [11] (see below). Both bumps, G09-bump and H11-bump, are generated with "back-leg-windings" on certain AGS main magnets.

Each of the "back-leg-windings", is powered by a "half sine-wave" current pulse which reaches its amplitude in $\sim 3.5 \mathrm{msec}$, and its action on the beam is a horizontal bend proportional to the current of the "back-leg-winding".

The location of these "back-leg-windings" has been chosen to cause one single $\lambda$ orbit bump at the vicinity of the G10 straight section and another $\lambda$-orbit bump at the vicinity of the H10 straight section (see fig 9).

A detailed description on the location, and strength of the "back-leg-windings", as well as on the orbit deformation which is caused by these bumps, is given in Ref. 12 . Figure 10 shows a schematic diagram of the FEB section of the AGS showing the location of the G10-kicker (FKG10) the location of the H10-septum (SMH10), the location of the magnets which generate the "local-bumps", the orbit of the circulating "bumped-beam", (note the "G09" and "H11" bumps), and the trajectory of the extracted beam into the ATR transfer line.

b) "Kick" the bunch with the G10-kicker magnet.

The action of the G10-kicker is to "kick" one bunch of the beam (provide and angular displacement of $\sim 1.6 \mathrm{mrad}$ of the beam) thus displacing the bunch at the location of

5 The beam from the AGS was extracted one bunch at a time and at time intervals of $33.33 \mathrm{msec}$ form bunch to bunch (see text below). 
the H10-septum which is located at $\sim 260^{\circ}$ phase advance from the kicker, and move the bunch to the other side of the septum (high field region).

In the remaining of the section we describe the geometry of the kicker and the "kicking" proccess.

Just after the G09 and H11 local orbit bumps have been created, the beam bunches pass through the center of the aperture of the G10 kicker magnet [11] which is located at the SS-G10 straight section of the AGS.

The center of the G10 kicker's aperture is located at $\sim 60 \mathrm{~mm}$ from the ideal orbit, and the inside edge of the kicker is located $\sim 44 \mathrm{~mm}$ from the ideal orbit of the circulating beam in the AGS, thus providing an unobstructed vertical aperture of $22 \mathrm{~mm}$ over the $32 \mathrm{~mm}$ horizontal aperture of the kicker.

Table 1A provides the beam parameters which determine the horizontal beam size ${ }^{6}$ at the middle of the G10 kicker, it also provides the location of the edges of the G10 kicker and the location of the beam centroid before and after the G10 kick.

The vertical beam size at the middle of the G10 kicker $\left(\beta_{x}=\beta_{y}=15 \mathrm{~m}\right)$ is $\sim \pm 5 \mathrm{~mm}$.

Table 1A

\begin{tabular}{|c|c|c|c|c|c|c|c|}
\hline \multicolumn{4}{|c|}{$\begin{array}{c}\text { AGS Beam Param. at the middle of } \\
\text { G10-kicker with Bumps ON. }\end{array}$} & \multicolumn{2}{c|}{$\begin{array}{c}\text { kicker-edge } \\
\text { from OCO [mm] }\end{array}$} & \multicolumn{2}{c|}{$\begin{array}{c}\mathrm{x}_{\text {cod }} \text { from OCO } \\
{[\mathrm{mm}]}\end{array}$} \\
\hline$\beta_{\mathrm{x}}[\mathrm{m}]$ & $\eta_{\mathrm{x}}[\mathrm{m}]$ & $\delta \mathrm{p} / \mathrm{p}$ & $\begin{array}{c}\text { Beam-size }[\mathrm{mm}] \\
\varepsilon=40 \pi \mathrm{mm} . \mathrm{mrad}\end{array}$ & Inner & Outer & $\begin{array}{c}\text { Before } \\
\text { kick }\end{array}$ & $\begin{array}{c}\text { After } \\
\text { kick }\end{array}$ \\
\hline 15 & -1.58 & 0.002 & \pm 5.8 & 44 & 76 & 60 & $\sim 60$ \\
\hline
\end{tabular}

Table 1A: Beam parameters $\left(\beta_{\mathrm{x}}, \eta_{\mathrm{x}}, \delta \mathrm{p} / \mathrm{p}\right.$ and $\left.\varepsilon\right)$ that determine the Horizontal beam size (column 4) at the middle of the G10 kicker magnet. The locations of the inner and outer edges of the G10 kicker magnet (column 5,6) at the center of the SS-G10 and the center of the bunch at the middle of the SS-G10 just before and after the G10 kick (column 7,8) are in reference to the OCO (Optimum Closed Orbit).

This transverse displacement of the kicker from the central orbit prevents any interaction of the beam bunches with the kicker while the beam bunches circulate at injection energy and have a size larger than the vertical kicker gap of $\sim 22 \mathrm{~mm}$.

When the beam is accelerated to a momentum of $24.02 \mathrm{GeV} / \mathrm{c}$, the beam size is reduced to $\pm 5 \mathrm{~mm}$ and therefore can fit into the aperture of the kicker when the bunch is locally bumped by the G09-bump.

A "full-aperture" G10-kicker would not require a G-bump but a larger aperture (gap) kicker that would accommodate the beam size at injection, would require a much larger power supply to provide the necessary kick to extract the beam from the AGS machine.

At any given time when the beam bunches pass through the aperture of the G10kicker the kicker may be energized to kick a single bunch out of its closed orbit, and direct it into the extraction channel of the H10-septum (see next).

6 The beam size corresponds to a $95 \%$ beam emittance that contains $95 \%$ of the beam intensity.

7 The locations are in reference to the OCO (Optimum Closed Orbit) which is the ideal beam orbit in the AGS. An ideal orbit is one that passes through the center of the quadrupoles thus no betatron oscillations are induced. 
The current pulse which powers the G10-kicker is a "half-sine" with T/2 400 nsec. An oscilloscope trace of the current pulse that energizes the G10 kicker, is shown in figure 12. The smallest spacing between two consecutive circulating bunches is $\sim 225$ nsec, (this is shown by the oscilloscope trace labeled "Bunch-current" in figure 12) therefore the kicker kicks only a single bunch during the time it is activated (400 nsec) This single bunch extraction assumes that the bunch is "kicked" by the G10kicker when the G10-kicker is at its maximum field strength and this occurs when the bunch is "properly synchronized" (see below) with the firing time of G10-kicker. The frequency $\mathrm{f}_{\text {kick }}$ that the $\mathrm{G} 10$ kicker can be activated is $\sim 60 \mathrm{~Hz}(17 \mathrm{msec})$. However the firing frequency of the $\mathrm{H} 10$ septum is $\mathrm{f}_{\text {septum }} \sim 30 \mathrm{~Hz}(33.33 \mathrm{msec})$, therefore the firing frequency of the septum magnets is the limiting factor of the AGS extraction frequency ( $f_{\text {extr }}=30 \mathrm{~Hz}$ ) or every $33.33 \mathrm{msec}$. A DC septum will increase the extraction frequency to that of the G10 extraction kicker.

c) Extract the bunch from the AGS using the H10-septum magnet.

The function of the H0-septum is to extract the beam from the AGS ring, after the beam is kicked by the G10-kicker (see section b above) into the high field region of the septum. The rest of the section describes the geometry of the H10-septum and its function during the beam extraction.

During the time the G09 and H11 bumps provide maximum displacement of the bunches and just before the G10-kicker fires, the bunches are circulating through the aperture of the G10-kicker and also pass $\sim 11 \mathrm{~mm}$ near the inner edge of the septum of the H10-magnet whose inner edge is located at $\sim 62.5 \mathrm{~mm}$ from the ideal orbit. Table 1B provides the beam parameters which determine the beam size at the entrance of the H10 septum, it also provides the location ${ }^{8}$ of the septum edges (columns 5,6) and the location/direction of the beam centroid (columns 7,8) before and after the G10 kick at the entrance of the H10-septum magnet.

Table 1B

\begin{tabular}{|c|c|c|c|c|c|c|c|}
\hline \multicolumn{4}{|c|}{$\begin{array}{c}\text { AGS Beam Param. at } \\
\text { H10-septum Entrance with Bumps ON. }\end{array}$} & \multicolumn{2}{c|}{$\begin{array}{c}\text { Septum-edge } \\
\text { from OCO [mm] }\end{array}$} & \multicolumn{2}{c|}{$\begin{array}{c}\mathrm{x}_{\text {cod }}, \mathrm{x}^{\prime} \text { cod from } \\
\text { OCO [mm,mrad] }\end{array}$} \\
\hline$\beta_{\mathrm{x}}[\mathrm{m}]$ & $\eta_{\mathrm{x}}[\mathrm{m}]$ & $\delta \mathrm{p} / \mathrm{p}$ & $\begin{array}{c}\text { Beam-size }[\mathrm{mm}] \\
\varepsilon=40 \pi \mathrm{mm} . \mathrm{mrad}\end{array}$ & Inner & Outer & $\begin{array}{c}\text { Before } \\
\text { kick }\end{array}$ & $\begin{array}{c}\text { After } \\
\text { kick }\end{array}$ \\
\hline 17 & -1.424 & 0.002 & 5.9 & 62.5 & 72.5 & $51,-3$ & $81,-5$ \\
\hline
\end{tabular}

Table 1B Beam parameters $\left(\beta_{\mathrm{x}}, \eta_{\mathrm{x}}, \delta \mathrm{p} / \mathrm{p}\right.$ and $\left.\varepsilon\right)$ that determine the beam size (column 4) at the entrance of the septum magnet. The locations of the inner and outer edges of the septum magnet (column 5,6) and the center of the bunch at the entrance of the H10-septum before and after the G10 kick (column 7,8) are in reference to the OCO (Optimum Closed Orbit).

The vertical beam size at the entrance of H10 septum (where $\beta_{\mathrm{y}}=12 \mathrm{~m}$ ) is $\sim 4.4 \mathrm{~mm}$ (we assume that the vertical dispersion function $\eta_{y}=0$ ).

8 The locations are in reference to the OCO (Optimum Closed Orbit) which is the ideal beam orbit in the AGS. An ideal orbit is one that passes through the center of the quadrupoles thus no betatron oscillations are induced. 
After the beam bunch is kicked by the G10-kicker the bunch is displaced, at the entrance of the H10 septum, by an additional $\sim 30 \mathrm{~mm}$ to a distance $\sim 81 \mathrm{~mm}$ away from the ideal trajectory. The required strength of the G10 kicker, to accomplish this displacement of the beam by $\sim 30 \mathrm{~mm}$ at the H10 location, corresponds to an angular kick, from the G10-kicker, of $\sim 1.6 \mathrm{mrad}$ into the inside of the AGS ring .

Thus the kick of the G10 kicker displaces the trajectory of the kicked bunch across the H10-septum and well within the extraction channel of the H10-septum magnet which deflects the beam bunch out of AGS ring into the U-V-line which subsequently transports the beam bunch to the V-target.

The required strength of the H10 septum magnet which is required to direct the beam bunch along the U-line corresponds to an angular beam deflection of $\sim 20 \mathrm{mrad}$ to the outside of the AGS ring.

\section{B) The Time Sequence of the FEB "triggers" during Beam Extraction}

During the FEB extraction process, each of the bunches must be synchronized with both, the RF signal, which drives the acceleration cavities, and the current pulses, which excite the extraction devices mentioned in the previous subsection (A).

In this subsection (B) we describe the time sequence of the various triggers that are necessary to initiate a particular operation of each of the extraction devices during the FEB process.

a) The "FEB-Request" trigger.

This trigger initiates the extraction process of a single bunch from the AGS. There is an FEB-Request trigger for each of the bunches to be extracted from the AGS machine.

The first FEB-Request trigger is usually set after the completion of the acceleration cycle and each subsequent trigger is set at $33.333 \mathrm{msec}\left(\mathrm{f}_{\max }=30 \mathrm{~Hz}\right)$ later from the previous one.

This frequency $\left(\mathrm{f}_{\max }=30 \mathrm{~Hz}\right)$ currently is constrained by the charging time of the H10-septum power supply.

For this particular run of the g-2 experiment, the first FEB-Request has been set 1.4 sec from $\mathrm{T}_{0}{ }^{2}$.

Each FEB-request initiates the following events, which are summarized in Table 2.

TABLE 2

\begin{tabular}{|c|c|c|c|c|}
\hline Device & Event-Name & $\begin{array}{c}\text { Start-Chrg-event } \\
{[\mathrm{msec}]}\end{array}$ & $\begin{array}{c}\text { Stop-Chrg-event } \\
{[\mathrm{msec}]}\end{array}$ & $\begin{array}{c}\text { Fire-event } \\
{[\mathrm{msec}]}\end{array}$ \\
\hline & & & & {$[\mathrm{msec}]$} \\
\hline G09-bump & FEB.G09A/B & 10 & & $\sim 24$ \\
\hline H11-bump & FEB.H11A/B & 10 & & $\sim 24$ \\
\hline G10-kicker & FEB.FKG10 & 15 & 25 & $28+$ Fine-delay \\
\hline H10-septum & FEB.SMH10 & 1 & & 26.9 \\
\hline
\end{tabular}

TABLE 2 Nomenclature and timing of the various events that occur during FEB

$9 \mathrm{~T}_{0}$ is the time when the AGS main-magnet-cycle is initiated. 
The trigger "events" in Table 2 are measured from time $\mathrm{T}_{0}$. These events are adjusted in time by the user, in order to synchronize the extracted bunches with the activation time of the extraction devices. The adjustment of all the time-events ("triggers") described in this section is done by the use of software applications which are readily available to the user.

\section{Start-Charge-event:}

a.1) of the power supplies 10 which power the "back-leg-windings" of G09-bump and H11-bump.

These events, which are 4 in number (one for each power supply), are set $\sim 10$ msec after the FEB-request.

a.2) of the power supply of the G10 kicker.

This event is set $\sim 15 \mathrm{msec}$ from the FEB-request and is followed by a stop charging event of the G10 kicker power supply. The stop-charging-events is set $\sim 25 \mathrm{msec}$ from the FEB-request.

a.3) of the power supply of the H10 septum magnet. This event is set $\sim 1 \mathrm{msec}$ from the FEB-request.

Firing-events:

b.1) of the G09-bump and H11-bump.

There are four fire events for the bumps; two fire events for the G09-bump (A/B power supplies ) and two for the H11-bump (A/B power supplies). Each of the events has been set at $\sim 24 \mathrm{msec}$ after the FEB-request.

Adjustment of the "fire events" is based on two requirements:

First the current amplitude of the power supply for the G09A "back-legwindings" should occur at the same time with the current amplitude of the power supply for the G09B "back-leg-winding". Similarly the current amplitudes of the power supplies for the H11A and H11B "back-leg-windings" should reach their amplitude simultaneously.

The second requirement, which is accomplished by adjusting the "firing time", is the simultaneous maximum beam displacement 11 at the straight sections G08 / G12 and H08 / H12 as recorded by the BPM's of the AGS-orbit program. Figure 12 shows clearly that the maximum displacement of the beam ${ }^{11}$, shown by the trace labeled "G08 HOR.BPM", occurs later in time than the maximum of current in the H11A/B bumps, shown by the traces labeled "H11A", "H11B".

b.2) of the H10 septum magnet.

10 There are two power supplies that power the "back_leg_windings" of the G-bump (A and B power supplies), and two power supplies that power the "back-leg windings"of the H-bump (A and B power supplies).

11 Note in Fig. 10 that the amplitude of the current that powers the "back-leg-winding" (trace labeled H11B) is not exactly in phase with the amplitude of the beam (trace labeled G08) at the location of the bumps. This may be due to the eddy currents formed on the AGS magnet chamber and on the main magnet coils when the "back-leg-windings" of the bumps fire. These eddy currents reduce and delay the maximum field generated by the bumps. 
This event occurs at $\sim 26.9 \mathrm{msec}$ from the FEB-request and it is adjusted so that the maximum magnetic field in the $\mathrm{H} 10$ septum, and therefore the maximum deflection of the extracted bunch, is synchronized with the maximum beam displacement generated by the G09 and H11 bumps.

This synchronization of the maximum magnetic field at $\mathrm{H} 10$ with the maximum beam displacement generated by the G09 and H11 bumps is verified by looking at the bunch deflection recorded by the first BPM (uhb1) of the U-V-line (the beam deflection at the uhb1 should be at a maximum). The "timing adjustments" discussed in b.1) and b.2) above are summarized in figure 12 .

Figure 11 (top) is an oscilloscope trace of the "back-leg-windings" currents of the G_bump (G09A) and the H_bump (H11A, H11B) and the current of the H10 septum.

Figure 11 (bottom) shows also the horizontal displacement of the beam as measured by the G08_BPM. Note that the maximum beam displacement as measured by the G08_BPM, occurs at a later time $(\sim 0.5 \mathrm{msec})$ than the maximum current in the "back-leg-windings".

The firing time of the $\mathrm{H} 10$ septum is adjusted to yield maximum beam displacement at the first BPM of the U-line. The vertical line ${ }^{12}$ in figure 10 (top and bottom) which runs through the maximum of current pulse of the H10 magnet, coincides with the time that the G10 kicker fires.

\section{b.3) of the G10 kicker}

After the firing of the G09-bump and H11-bump the bunches circulate in the AGS and are locally bumped (see Figures 9,10) at the vicinity of SS-G10 and SS-H10 straight sections.

When the amplitude of the bumped beam and the B-field amplitude of the H10septum is reached, the ARF.FEBREQ.DELAY trigger is generated at $\sim 28 \mathrm{msec}$ after each FEB-request trigger.

The ARF.FEBREQ.DELAY trigger ${ }^{13}$ energizes a circuit that comes under the name "kicker trigger generator" which performs the following functions:

a) Just after the arrival of the ARF.FEBREQ.DELAY, the "kicker-triggergenerator" synchronizes the AGS bucket (of the first injected bunch from the AGS-Booster), with the immediate next "zero-crossing" of the RF signal which is also fed into the "kicker trigger generator" circuit.

This synchronization of the "zero-crossing" of the RF signal is required in order to keep track of the extracted bunches in the AGS.

b) Generates a trigger which comes under the name "G10-kicker-trigger". The "G10-kicker-trigger" is synchronized also with the AGS bucket of the first injected bunch from the AGS-Booster.

The synchronization the AGS bucket of the first injected bunch from the AGS-Booster with the "zero-crossing" of the RF signal and the "G10-kicker-trigger" is accomplished by

12 This vertical line is noise which is picked-up by the signal-cables when the G10-kicker fires.

13 Detailed information about the signal processing following the ARF.FEBREQ.DELAY trigger and the circuitry involved can be found in ref. [17,18] 
means of an additional signal First-Transfer-Marker(FXM) which is generated from the AGSBooster RF, and fed also into the "kicker trigger generator" circuit.

After the extraction of the first bunch from the AGS, the "kicker-trigger- generator" synchronizes the next AGS bucket with the "zero-crossing" of the RF signal and the "G10kicker-trigger", until all bunches are extracted from AGS.

Before the extraction of a particular bunch from the AGS, the "G10-kicker-trigger" signal is fed into another circuit $[17,18]$ which generates the "FEB-bunch trigger" 14 which causes the G10kicker to fire after a time delay (known as "fine delay") from the "FEB-bunch trigger".

The purpose of this "fine delay" is to synchronize the bunch with the amplitude of the B-field generated by the G10-kicker, so that the bunch receives the maximum kick when it passes through the G10-kicker. This fine delay once adjusted by the user, remains the same for all extracted bunches.

The "FEB-bunch trigger" is sent also to the "RHIC EVENT LINK" which generates an event known as "FEB bunch". The "FEB bunch" is sent to the circuitry of other devices like BPM's, current-transformers, and loss monitors of the AtR line.

A similar delay, like the "fine-delay" which is applied to the "FEB-bunch trigger", for synchronization of the extracted bunch with the firing of the "G10-kicker", must also be applied to the "FEB bunch" for synchronization of the BPM's, current-transformers, and loss monitors of the AtR line with the extracted bunches [17].

In the rest of this section we will provide some information on various current pulses which were recorded by the digital oscilloscope and are associated with the fast extraction process.

The two oscilloscope traces which are shown in figure 12 and labeled as "Bunch-Current" correspond to the longitudinal charge distribution of the beam bunches as measured by the "wall current monitor". The top trace with the negative spikes is displayed on a time-base of $500 \mathrm{nsec}$ and the bottom trace is displayed on a time base of $50 \mathrm{nsec}$. The base to base bunch length is $\sim 80$ nsecs.

The positive "half-sine-wave" curve in the graph labeled "G10 current pulse" corresponds to the kicker current which is displayed in $50 \mathrm{nsec} / \mathrm{div}$ scale. The duration of the current pulse, as shown by the oscilloscope trace, is 400 nsec.

For high intensity proton operations the high beam current induces a significant voltage on the coil of the G10-kicker. This voltage, in turn, generates a noise[13] on the "G10-kicker trigger" pulse which causes an earlier firing of the kicker, than what is expected by the "fine delay". A time distribution of the G10 firing events, as measured with respect to the "G10-kickertrigger", is shown in figure 13.

This figure shows that there is a "total beam charge, 15 dependence, of the time difference between the "G10-kicker-trigger", and the "G10 kicker firing" time. This "total beam charge" dependence does not allow the user to bring each bunch in the G10 kicker aperture when the kicker is at its maximum strength.

14 This trigger is also sent to the RHIC EVENT LINK timing system, under the code known as "FEB-bunch".

15 The expression "total beam charge" is referred to the total charge of the circulating beam. 
This "time misalignment" affects the radiation levels (see fig.14) in the extraction region during the extraction of the last two bunches that are not timed correctly with respect to the "kicker firing".

A timing system that allows the user to set a different "fine delay" for each extracted bunch will improve this "timing misalignment" between the bunches and the G10-kicker firing.

\section{Beam Optics of U-V line}

The extracted beam-bunches from the AGS were transported via the U-V-line [3] to the V-target [1]. The beam optics of the U-V line is based on the following items.

a) The beam parameters at the beginning of the U-V line.

b) The beam optics of the U-V line.

c) The beam parameters at the beginning of the U-V line depend on the optical parameters (tunes, chromaticities, extraction settings) of the AGS ring during extraction.

In this section we discuss the items (a) to (c) above(not in same order). We also discuss the effect of the error in the strength of the G10-kicker and of the H10-septum on the U-V line beam optics.

\section{a. Optical parameters of the AGS ring during extraction}

The optics of the AGS and other relevant parameters that have been used to calculate the beam parameters at the origin of the U-V-transfer line, during beam extraction, are shown in TABLE 3.

TABLE 3

\begin{tabular}{|c|c|c|c|c|c|c|c|c|c|}
\hline & $\begin{array}{c}\mathrm{P}_{\text {ext }} \\
{[\mathrm{GeV} / \mathrm{c}]}\end{array}$ & $\mathrm{Q}_{\mathrm{x}}$ & $\mathrm{Q}_{\mathrm{y}}$ & $\begin{array}{c}\mathrm{I}_{\mathrm{Qx}} \\
{[\mathrm{A}]}\end{array}$ & $\begin{array}{c}\mathrm{I}_{\mathrm{Qy}} \\
{[\mathrm{A}]}\end{array}$ & $\xi_{\mathrm{x}}$ & $\xi_{\mathrm{y}}$ & $\begin{array}{c}\mathrm{I}_{\mathrm{Sx}} \\
{[\mathrm{A}]}\end{array}$ & $\begin{array}{c}\mathrm{I}_{\mathrm{Sy}} \\
{[\mathrm{A}]}\end{array}$ \\
\hline set & 24.02 & 8.76 & 8.75 & 266 & -212 & -2.78 & 0.66 & 94 & 150 \\
\hline calc & 25.63 & 8.77 & 8.79 & 266 & -212 & -2.49 & 1 & -94 & 150 \\
\hline
\end{tabular}

TABLE 3: The "set" and calculated optical parameters of the AGS synchrotron during fast beam extraction.

The symbol $\mathrm{P}_{\text {ext }}$ in column 2 of TABLE 3 corresponds to the beam momentum during extraction. This establishes the magnetic field generated by the main AGS magnets for a given radius of extraction.

The symbols $\mathrm{Q}_{\mathrm{x}}, \mathrm{Q}_{\mathrm{y}}, \mathrm{I}_{\mathrm{Qx}}, \mathrm{I}_{\mathrm{Qy}}$ in column 3 to 6 are the horizontal and vertical tunes of the AGS synchrotron during extraction and the currents of the horizontal and vertical tune quadrupoles. The values of both the horizontal and vertical tunes during the AGS magnet cycle appear in figure 3.

The symbols $\xi_{\mathrm{x}}, \xi_{\mathrm{y}}, \mathrm{I}_{\mathrm{Sx}}, \mathrm{I}_{\mathrm{Sy}}$ in column 7 to 10 are the horizontal and vertical chromaticities of the AGS synchrotron during extraction and the currents of the horizontal and vertical chromaticity sextupoles. 
The values of both the horizontal and vertical chromaticities during the AGS magnet cycle appear in figure 4.

Row 2 in TABLE 3 corresponds to the values "set by the user" (see figs. 3 and 4 ) and the row 3 corresponds to the calculated values using the code AGS_BATE (AGS Beam Acceleration, Transport and Extraction) [12] (see also APPENDIX 1).

No measurements of the tunes and chromaticities were performed during the $\mathrm{g}-2$ run.

\section{b. Beam parameters at the beginning of the U-V line}

The beam parameters at the straight section SS-H13 (beginning of U-V-line) as calculated using the settings of the optical functions of the AGS ring discussed above and appearing in TABLE 3 are shown in TABLE 4 below.

TABLE 4

\begin{tabular}{|c|c|c|c|c|c|}
\hline$\beta_{\mathrm{x}}[\mathrm{m}]$ & $\alpha_{\mathrm{x}}$ & $\eta_{\mathrm{x}}$ & $\eta^{\prime}{ }_{\mathrm{x}}$ & $\beta_{\mathrm{y}}[\mathrm{m}]$ & $\alpha_{\mathrm{y}}$ \\
\hline 30.8 & -3.32 & -1.15 & -0.08 & 8.0 & 1.18 \\
\hline
\end{tabular}

TABLE 4: The calculated beam parameters at the origin of the U-V-line for the AGS optics determined by the parameters shown in TABLE 3.

The procedure to obtain these beam parameters shown in TABLE 4 is described in Ref. [12] and is outlined in APPENDIX 1.

The beam parameters which appear in TABLE 3 are referenced to the same coordinate system that the MAD computed code is using.

There were no measurements performed during the $g$ - 2 experiment, for the determination of the beam parameters shown in TABLE 4.

\section{c. Beam optics of the U-V line}

The layout of the magnetic elements, which comprise the U-V line, are shown in APPENDIX 3 in the form of a TRANSPORT input-data file.

Figure 18 shows the horizontal (X) and vertical (Y) beam profiles of the beam in the U-Vline. The beam profiles correspond to a $95 \%$ normalized beam emittance $\varepsilon_{\mathrm{x}, \mathrm{y}}=100$ pi.mm.mrad.

\section{Effect of field errors and momentum errors}

Field errors (deviations of the field strength from their nominal value) of the G10 kicker and/or the H10 septum magnets and/or the G09,H11 Bumps, will cause a displacement of the central orbit $\left(\mathrm{x}_{\mathrm{cod}}, \mathrm{X}^{\prime}\right.$ cod $)$ in phase space, at any point along the $\mathrm{U}-\mathrm{V}$-transport-line, including the $\mathrm{V}$-target. The momentum variation $\Delta \mathrm{p}$ of the extracted beam will also have similar effect on the U-V-transport-line.

In order to determine the effect of the magnet errors and momentum variations on the central orbit displacement ( $\mathrm{x}_{\mathrm{cod}}, \mathrm{x}_{\mathrm{cod}}$ ), two different but equivalent methods (see APPENDIX 2) have been used. 
a) The first method is based on the use the AGS_BATE code which calculates the displacement of the central orbit ( $\mathrm{x}_{\mathrm{cod}}, \mathrm{X}^{\prime}{ }_{\mathrm{cod}}$ ) in phase space, (at the origin of the U-Vtransport-line), due to the variation of the extraction devices and beam momentum. These results appear in TABLE 5:

In this table the convention, on the coordinate-system used and on the sign of the kicker, or septum strength, is the same as that in the computer code MAD.

For example (column 2 in Table 5) positive kick angle in G10 kicker results in a negative displacement in position and angle in the H13 location.

Similarly (column 4 in Table 5) an increase in the G10 bump strength results in a negative displacement in position and angle in the H13 location (origin of U-V-line). APPENDIX 2 provides a description on how to calculate the central orbit displacement $\left(\mathrm{x}_{\text {cod }}, \mathrm{x}_{\text {cod }}\right)$ in phase space, at any point along the $\mathrm{U}-\mathrm{V}$-transport-line using the data in TABLE 5.

TABLE 5

\begin{tabular}{|c|l|l|l|l|c|}
\hline Effect & G10 kicker & H10 septum & G09 Bump & H11 Bump & $\Delta \mathrm{p} / \mathrm{p}$ \\
\hline$\Delta \mathrm{x}_{\text {cod }}[\mathrm{mm} / \%]$ & -0.364 & 2.965 & 0.04 & -0.8 & -11.495 \\
\hline$\Delta \mathrm{x}_{\text {cod }}[\mathrm{mrad} / \%]$ & -0.0323 & 0.425 & 0.0009 & -0.08 & -0.62 \\
\hline
\end{tabular}

TABLE 5: The effect of the extraction devices (G10 kicker, H10 septum, G10/H10 Bumps) and beam momentum variations on the phase space coordinate ( $\mathrm{x}_{\text {cod }}, \mathrm{X}^{\prime}$ cod $)$ of the central trajectory, at the location of the origin of the U-V-line. The variations $\Delta \mathrm{x}_{\text {cod }}$ and $\Delta \mathrm{x}^{\prime}$ cod (rows 2 and 3 ) correspond to $1 \%$ variation in the strength of the extraction devices or $1 \%$ in $\Delta \mathrm{p} / \mathrm{p}$

Figure 19 plots the theoretical and experimental data of the central orbit displacement $\left(\mathrm{x}_{\mathrm{cod}}\right)$ of the last extracted $\left(12^{\mathrm{th}}\right)$ bunch along the $\mathrm{u}-\mathrm{v}$ line. This central orbit displacement is generated by varying the strength of the G10-kicker by $\pm 10 \%$.

The theoretical data (solid and dotted lines in Fig. 19) were calculated using the method described in this subsection (a). The experimental data (filled circles in Fig. 19) were obtained using the readings of the available BPM's of the $\mathrm{u}-\mathrm{v}$ line. The error bars of the experimental points are mainly due to systematic errors on the G10-kicker strength and/or G10-kicker "timing"16

The errors in the predictions coming from these variations can be corrected by measuring the strength and "timing" of the G10-kicker. The errors introduced by the BPM's are smaller than $1 \mathrm{~mm}$. The non-agreement of the theoretical and experimental data shown in Fig. 19, is mainly due to the disagreement of the theoretical predicted values of the $\mathrm{x}_{\mathrm{cod}}$ and $x^{\prime}$ cod with the actual values, at the beginning of the U-V-line.

These quantities ( $\mathrm{x}_{\text {cod }}$ and $\mathrm{x}_{\text {cod }}$ ) depend on the optics of the AGS. Indeed by varying $\mathrm{x}_{\text {'cod }}$ at the beginning of the U-V-line the agreement of the theoretical and experimental data falls within the experimental error.

Similar comparison theoretical and experimental data of the central orbit displacement generated by varying the strength of the H10-septum by $\pm 0.83 \%$ is shown in Fig. 20 .

16 Variations of the time difference between the time of G10-kicker trigger and the G10-kicker firing. 
Comparison of the data in Fig. 19 and Fig. 20, shows that the $\mathrm{x}_{\mathrm{cod}}$ is by an order of magnitude more sensitive to the variations of the H10-septum strength rather, than the variation of the strength of the G10-kicker strength.

b) The second method makes use of the first order transfer Matrices that describe the beam transport in the following sections in the AGS synchrotron:

- The section defined between the middle points of the G10 and H13 straight section of the AGS and

- The section defined between the middle points of the H10 and H13.

These matrices, which are also calculated using the AGS-BATE computer code, could also provide the same information (see APPENDIX 2), as in (a) above, on the displacement of the central orbit $\left(\mathrm{x}_{\mathrm{cod}}, \mathrm{X}^{\prime}{ }_{\text {cod }}\right)$ in phase space, at any location along the $\mathrm{U}$ $\mathrm{V}$-transport-line when the extraction devices are out of tune.

The relevant beam transport matrices, as calculated using the computer code AGS_BATE follow the notation:

\begin{tabular}{lcccc}
\multicolumn{3}{c}{ Horizontal } & \multicolumn{2}{c}{ Vertical } \\
$\mathrm{R}_{11}$ & $\mathrm{R}_{12}$ & $\mathrm{R}_{16}$ & $\mathrm{R}_{33}$ & $\mathrm{R}_{34}$ \\
$\mathrm{R}_{21}$ & $\mathrm{R}_{22}$ & $\mathrm{R}_{26}$ & $\mathrm{R}_{43}$ & $\mathrm{R}_{44}$ \\
0 & 0 & 1 & &
\end{tabular}

and appear below. The relevant units of the matrix elements are $[\mathrm{cm}]$ for space [mrad] for angle and $[\%]$ for $[\delta \mathrm{p} / \mathrm{p}]$.

Transfer matrices from G10 to $\mathrm{H13}$

\begin{tabular}{ccccc}
\multicolumn{3}{c}{ Horizontal } & \multicolumn{2}{c}{ Vertical } \\
-1.2945 & -21.599 & -2.17 & 1.2018 & -8.7255 \\
-0.072191 & -1.9769 & -0.139 & -0.14739 & 1.9023 \\
0.0 & 0.0 & 1.0 & &
\end{tabular}

Transfer matrices from G10 to H10

\begin{tabular}{ccccc}
\multicolumn{3}{c}{ Horizontal } & \multicolumn{3}{c}{ Vertical } \\
-1.6895 & -16.953 & -2.595 & 1.1586 & -13.616 \\
0.1776 & 1.1905 & 0.248 & 0.18345 & -1.2928 \\
0.0 & 0.0 & 1.0 & &
\end{tabular}

Transfer matrices from $\mathrm{H10}$ to $\mathrm{H13}$

\begin{tabular}{lllll}
\multicolumn{3}{c}{ Horizontal } & \multicolumn{2}{c}{ Vertical } \\
2.2955 & 14.545 & 0.148 & 0.046997 & 6.2541 \\
0.26523 & 2.1162 & 0.014 & -0.15841 & 0.19705 \\
0.0 & 0.0 & 1.0 & &
\end{tabular}

An upgrade version of the BPM application of the ATR line, will use data of the central orbit displacement $\left(\mathrm{x}_{\mathrm{cod}}\right)$, (measured by the BPM's, and will provide information on the deviation of the extraction devices (G10-kicker, H10-septum, G09/H11-Bumps) and of the beam momentum deviation $(\Delta \mathrm{p})$ from their nominal values. 


\section{A procedure to tune the settings of the extraction devices of the AGS synchrotron to minimize the radiation due to beam losses during extraction .}

A single extracted beam bunch from the AGS may contain $\sim 5 \times 10^{12}$ protons/bunch therefore only a small percentage of beam loss can be a potentially big source of radiation. One of the responsibilities of the AGS personnel is to comply with the ALARA program which is practiced by BNL and calls for minimization of the radiation caused by beam losses on the various accelerator components. In order to comply with the ALARA program, a procedure has been implemented to minimize the beam losses during the process of the fast extracted beam. In this section we identify the regions of the AGS where beam losses may occur during fast extraction ${ }^{17}$ and discuss the procedure used for the minimization of the beam losses.

The possible regions where radiation may be generated during beam extraction are:

1. The region along the SS-G10 straight section where the G10 fast kicker is located. In this region (SS-G10) the beam is "locally bumped" in order to be placed well within the aperture of the G10 kicker.

The relatively small useful horizontal aperture of $\sim 25 \mathrm{~mm}$ and the small vertical gap of $22 \mathrm{~mm}$ of the G10 kicker in combination with the beam size $(\sim \pm 5.5$ and $\sim \pm 5.5 \mathrm{~mm}$ for the hor. snd ver. respevtivly at $95 \%$ intensity and $\varepsilon=40 \pi$.mm.mrad) makes the kicker-beam interaction very sensitive to beam misalignments.

The radiation at this region can be detected by the Beam Loss Monitors (BLM's) which are located at the SS-G10 region, and can be viewed by the AGS Loss Monitors application.

There are two additional radiation monitors at the SS-G10 region; one upstream of the G10 kicker and one downstream of the G10 kicker. The readings of the radiation detected by these monitors as well as their difference (Downstream_value - Upsteam_value) can be displayed on the "XBAR" oscilloscopes through the XBAR application.

For equipment protection an upper limit of the radiation at the G10 location is set; once this limit is exceeded the beam in the AGS is aborted to the J10 beam dump.

2. The region along the SS-H10 region where the extraction septum is located.

In this region (SS-H10) the beam while circulating is "locally bumped" close to the septum region of the H10 septum magnet. When the beam is "kicked" with the G10 kicker the beam is again in proximity to the H10 septum but on the other side of the septum and in the extraction channel of the H10 septum magnet. In either case the proximity of the beam bunch to the septum may be the cause of beam loss and a source of radiation. The BLM's at the location of the SS-H10 can record the radiation due to beam losses at this region, and the value of the radiation can be displayed either by the AGS beam loss application or on the XBAR oscilloscope.

3. The region after the H10 septum magnet and along the trajectory of the extracted beam.

17 Beam losses along the U-V transport line is of great concern. These losses have been minimized by steering the beam and observing the radiation monitors placed along the line. Ref[19] reports on the beam losses and minimization of beam losses at the region of the VQ12 quadrupole. 
This radiation is detected by the BLM's of the AGS and displayed by the AGS beam loss application.

A procedure to minimize the beam losses at the locations mentioned above is described below.

1. Just before extraction (the beam is locally bumped at the maximum displacement but the G10-kicker has not fired yet) we adjust:

a) the radial steering function.

b) the strength of the G10 and H10 bumps

We observe the AGS Beam Loss application display, shown in figure 14, for minimum radiation levels at the location of the SS-G10 and SS-H10. In addition we observe the radiation levels of the radiation monitors at G10 and H10 locations, as displayed by the XBAR oscilloscope and shown in figure 15.

2. At extraction (when the G10-kicker fires) we observe radiation levels with the monitors mentioned in part 1 above.

We adjust the G10-kicker and H10-septum strengths and possibly G10 and H10 bumps for minimum radiation levels.

3. We iterate steps 1 and 2 for minimum radiation levels.

Radiation levels recorded by the AGS Loss Monitor application, are shown in figure 14 . The XBAR oscilloscope display shown in figure 15 , plots the radiation levels as a function of time, during beam extraction, of the radiation detected by the BLMH10/Downstream the G10/Difference. The third curve on this plot is the circulating beam current in the AGS.

During the minimization procedure of the radiation levels we also observe the BPM's of the U-V-line for optimum beam trajectory shown in figure 16, and minimum radiation levels along the U-V-line, shown in figure 16.

\section{Conclusions}

The delivery of high intensity proton beam on the V-target during the g-2 experiment in the year 2000 was within normal operational limits as specified by the ALARA. The beam optics of the AGS synchrotron during extraction was tested using the U-V transport line. Further studies will enable a more accurate determination of the AGS optics during extraction. Specific modifications mentioned in this technical note will further improve the fast extraction beam operations of delivering beam into the $\mathrm{V}$-target.

18 The amplitude of the bump is sensitive to the B power supply of the bump. The residuals are sensitive to the A power supply of the bump. 


\section{APPENDIX 1}

The procedure to calculate the beam parameters at the beginning of the $\mathrm{U}-\mathrm{V}$-line is outlined below.

1. The computer code used in the calculations comes under the code name AGS_BATE (AGS Beam Acceleration, Transport and Extraction). This code is a modified version of the code BEAM [14] with the following modifications:

1a. The particle orbits in the AGS synchrotron are calculated using measured magnetic fields of the AGS main magnets[15]. This enables to take into account the magnetic fields of the main magnets in the fringe regions which are located outside the circulating beam region. These fringe field regions are traced by the particles during either the Fast Extracted Beam (FEB) and Slow Extracted Beam (SEB) processes.

1b. The devices which are used during the extraction processes (FEB or SEB) were simulated and introduced into the computer code. These devices generate the appropriate "local beam bumps" and "beam kicks" necessary for the beam extraction.

1c. Include into the code minimization/optimization routines which optimize the strength of the magnets that control the "local beam bumps" to achieve the proper beam amplitude, and also minimize the "central orbit beam residuals" 19 which occur outside the "local beam bumps".

1d. Capabilities of raytracing a set of particles with initial conditions read either from an input file or a normal (Gaussian) distribution.

1e. Computation of dispersion and chromaticities in the AGS.

2. Energize the appropriate magnets to generate the "local bumps" and optimize their strength for the desired beam amplitude at G10 and H10 locations, and minimization of the beam residuals during a closed orbit.

3. With a closed orbit established, adjust the strength of the G10 kicker, to displace the beam into the extraction channel of the H10 septum, and the strength of the H10 septum to direct the central orbit at the origin and the initial direction of the $\mathrm{U}-\mathrm{V}$-line.

4. The execution of steps 1 to 3 provides the necessary information for the calculation of the beam parameters [12] at the origin of the U-V-line.

19 The "central orbit beam residuals" are transverse beam oscillations that are generated outside the region of the main bumps, because the phase advance between the location of the magnets which generate the bumps is in general not the proper phase advance which eliminates these residuals. To minimize these residuals, the code optimizes the relative strength of the $\mathrm{A}$ and $\mathrm{B}$ bumps (see previous sections). 


\section{APPENDIX 2}

In this APPENDIX we outline two methods that can be used to calculate the variation of the phase space coordinates $\left(\mathrm{x}_{\mathrm{cod}}, \mathrm{X}^{\prime}\right.$ cod $)$ at any point along the $\mathrm{U}_{-} \mathrm{V}$-transport-line. Both methods are based on the equation A1 below.

$$
\left(\mathbf{X}_{\mathrm{cod}}\right)_{\text {out }}=\mathbf{R} \cdot\left(\mathbf{X}_{\mathrm{cod}}\right)_{\text {in }}
$$

The symbol $\mathbf{R}$ in Eq. A1 represents the first order transfer matrix which transforms the phase space coordinates $\left(\mathbf{X}_{\text {cod }}\right)_{\text {in }}=\left(\mathrm{x}_{\mathrm{cod}}, \mathrm{x}^{\prime}{ }_{\operatorname{cod}}, \Delta \mathrm{p}\right)_{\text {in }}$ at the entrance of a beam line to those coordinates $\left(\mathbf{X}_{\text {cod }}\right)_{\text {out }}=\left(\mathrm{x}_{\text {cod }}, \mathbf{x}^{\prime}{ }_{\text {cod }}, \Delta \mathrm{p}\right)$ out at the exit.

The $\mathbf{R}$ matrix can be calculated using the computer code TRANSPORT[16]. The input data file, which is used in the TRANSPORT calculations, is listed in APPENDIX 3 and contains the location, the geometrical parameters and magnetic strength, of the dipole and quadrupole magnetic elements of the beam line.

Method (a):

Using the TRANSPORT computer code calculate the $\mathbf{R}$ matrix that corresponds to the beam line starting at the origin of the U-V-transport-line (H13) to the point we need to calculate the deviation of the central trajectory $\left(\mathrm{x}_{\text {cod }}, \mathrm{x}^{\prime} \text { cod }\right)_{\text {out }}$.

Using TABLE 5 obtain values for the central orbit displacement $\left(\mathrm{x}_{\operatorname{cod}}, \mathrm{x}^{\prime}{ }_{\text {cod }}, \Delta \mathrm{p}\right)_{\text {in }}$ at the origin (H13) of the U-V-transfer-line that corresponds to a given deviation of the strength of the extraction devices from their nominal values.

Use equation $\mathrm{A} 1$ to obtain values for $\left(\mathrm{x}_{\mathrm{cod}}, \mathrm{x}^{\prime}{ }_{\text {cod }}\right)_{\text {out }}$.

Method (b):

Using the TRANSPORT computer code calculate the $\mathbf{R}$ matrix that corresponds to the beam line starting at the origin of the U-V-transport-line (H13) to the point we need to calculate the displacement of the central trajectory $\left(\mathrm{x}_{\mathrm{cod}}, \mathrm{x}^{\prime} \text { cod }\right)_{\text {out }}$.

To calculate the effect of the G10-kicker, multiply the $\mathbf{R}$ matrix above, with the $\mathbf{R}$ matrix which corresponds to the transport line starting from the G10 kicker to the beginning of the U-Vtransport-line.

Then calculate the effect of the deviation of the G10-kicker on the displacement of the central trajectory at the location of the G10-kicker. Such an effect is equivalent to an angular displacement $\left(0_{\text {cod }}, x_{\text {cod }}, 0\right)_{\text {in }}$.

Make use of equation A1 to obtain values for $\left(\mathrm{x}_{\mathrm{cod}}, \mathrm{x}^{\prime}{ }_{\mathrm{cod}}\right)_{\text {out }}$ at the desired location.

To calculate the effect of the H10-septum, the same procedure as for the G10-kicker above, is followed but the R matrix used corresponds to the beam line starting from H10 location to the beginning of the $\mathrm{U}-\mathrm{V}$-transport-line. 


\section{APPENDIX 3}

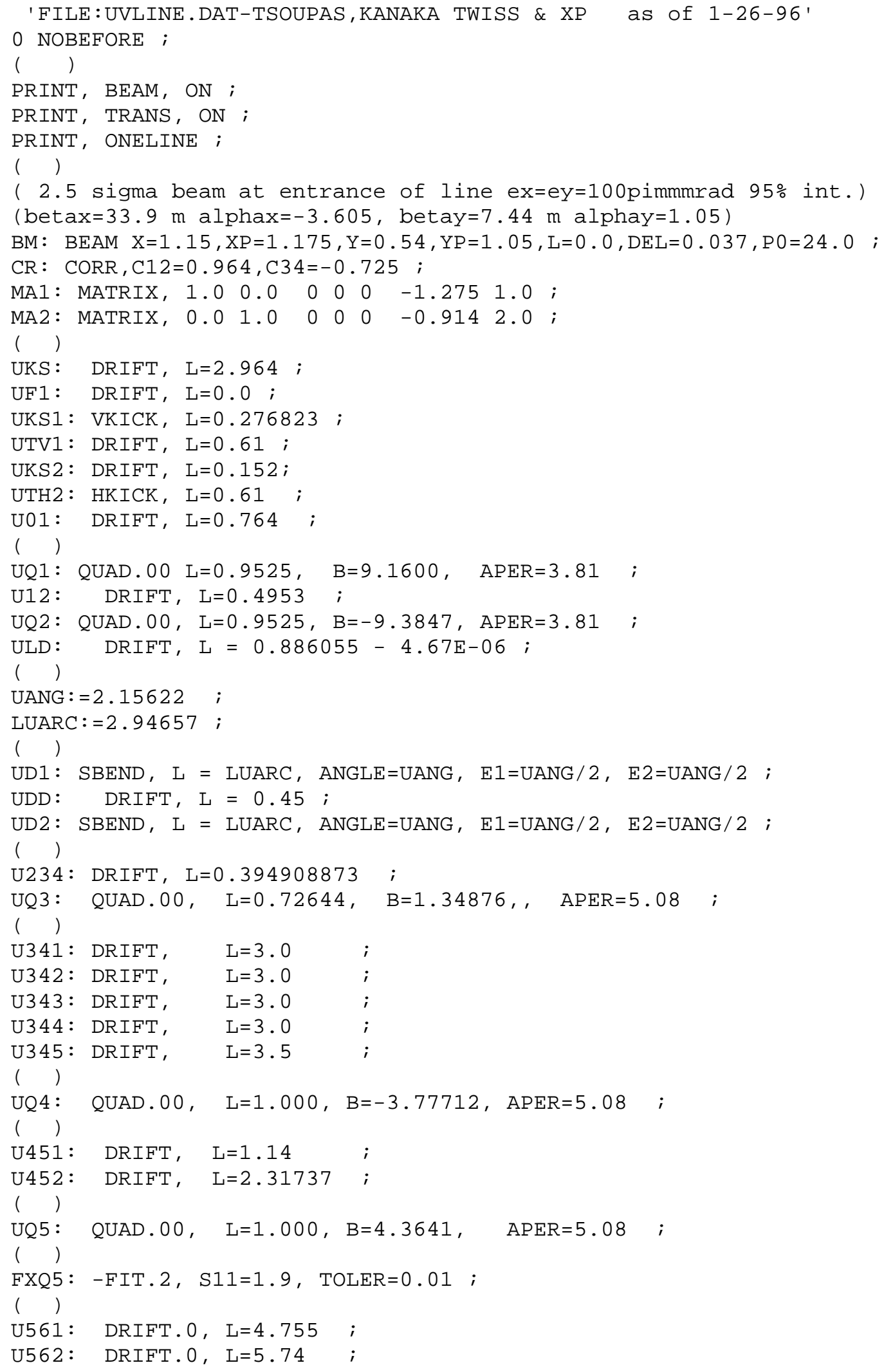




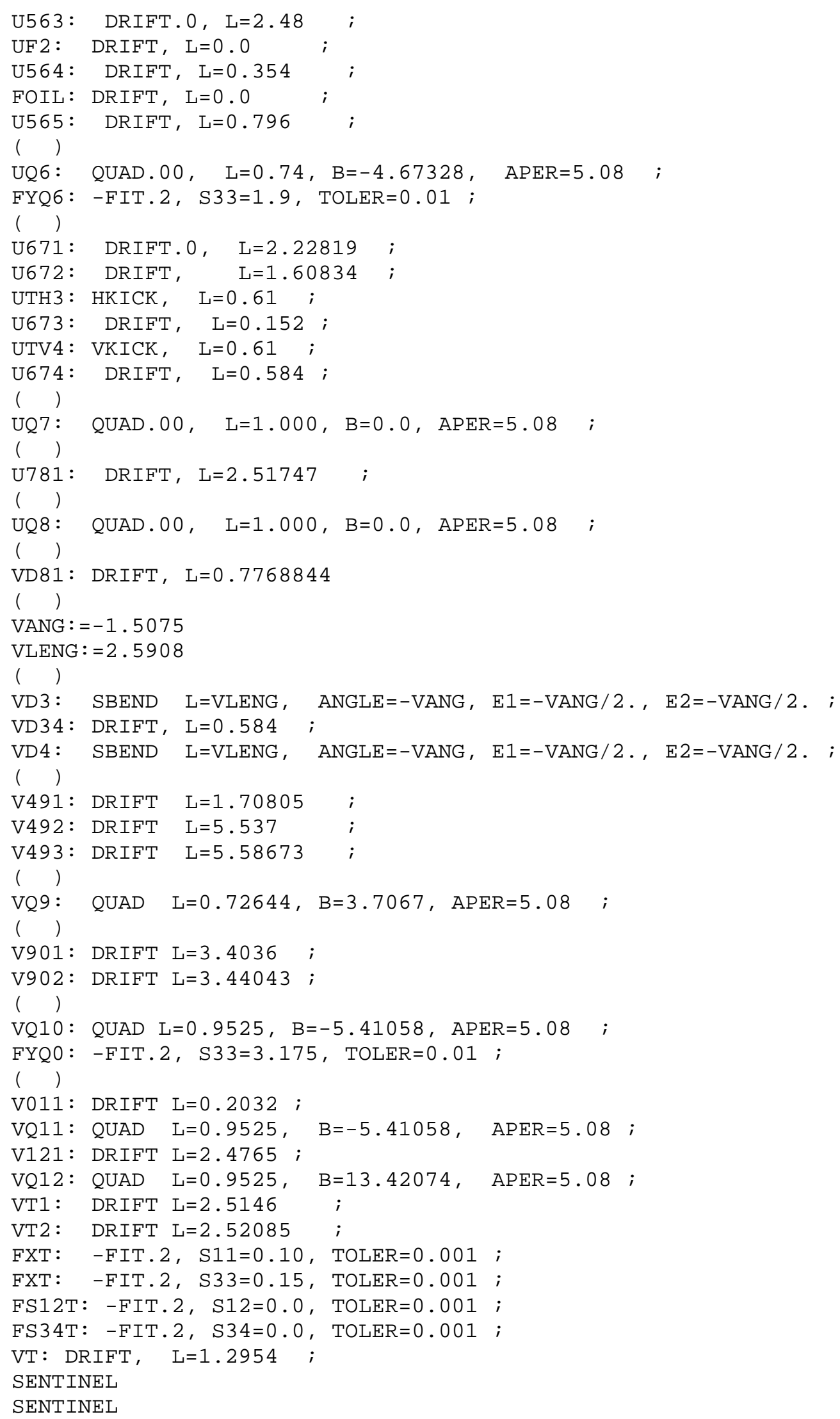




\section{REFERENCES}

[1] Design Report "A New Precision Measurement of the Muon (g-2) Value" BNL AGS E821 April 1994 page 73

[2] Design Report "A New Precision Measurement of the Muon (g-2) Value" BNL AGS E821 April 1994

[3] Design Report "A New Precision Measurement of the Muon (g-2) Value" BNL AGS E821 April 1994 page 72-75

[4] J. G. Alessi et. al. AIP Conf. Proc. P419 158.

[5] J. G. Alessi et. al. "Performance of the new RFQ Preinjector" p 999, PAC 1989

[6] S.S. Kurennoy et. al. Proc. Of the $19^{\text {th }}$ Int. Linac Conf. p1004 1998.

[7] G.W. Wheeler, et. al. PARTICLE ACCELERATORS, Vol. 9, Nos. 1,2 (1979)

[8] Booster Design Manual.

[9] N. Tsoupas, et al. "Tunes, Chromaticities and Dispersion of AGS, during the Fast Beam Extraction of Au for the operation of RHIC in the year 2000. Tech-note in preparation.

N. Tsoupas, et al. "Beam Parameters of the AGS Synchrotron during Fast Beam Extraction at the Location of the AGS Kicker" PAC Conf. June 2001.

[10] N. Tsoupas, W. Glenn BNL. Private Communication

[11] M. Tanaka "The New Fast Extraction System at the AGS AGS/AD/Tech. Note No. 347

[12] N. Tsoupas et. al. "Closed Orbit Calculations at AGS and Extraction Beam Parameters at $\mathrm{H} 13$ " $\mathrm{AD} / \mathrm{RHIC} / \mathrm{RD}-75$

[13] W. Zhang et. al. "Beam Coupling Phenomena in Fast Kicker Systems" PAC 2001 to be published.

[14] G. H. Morgan, "Fortran IV Version of Beam; The AGS Orbit Computing Program", AGS Internal Report.

E. D. Courant, "Computations of AGS Orbits with 704 Computer", ADD Internal Report EDC-36

[15] R. Thern, Experimental Magnetic Field Maps of the AGS Dipole Magnets, (Private Communication)

[16] D.C.Carey, et. al. "A Computer Program for Designing Charge Particle Beam Transport Systems. STANFORD LINEAR ACCELERATOR CENTER SLAC-R-95-462

[17] Leif Ahrens, "Timing and Gain Comments Relevent to the U,V,W,X, and Y, Line Current Transformers"

[18] http://www.cadops.bnl.gov/AGS/Operations/docum/documents/UVWXY_Xformer.html tttp://WwW.cadops.bnl.gov/Hardware/BNLonly/drawings/index.httml

[19] N. Tsoupas, G. Bunce "Determination of Maximum Allowed Radiation/Beam-Loss Limit at the Location of VQ12 Quadrupole of the V-line" Technote in preparation.

[20] V.V. Balandin et. al. "The Resonant Theory of Longitudinal Emittance Blow-up by Phase-Modulated High Harmonic Cavities" Particle Accelerators, V 35, p.1-14 (1991) 


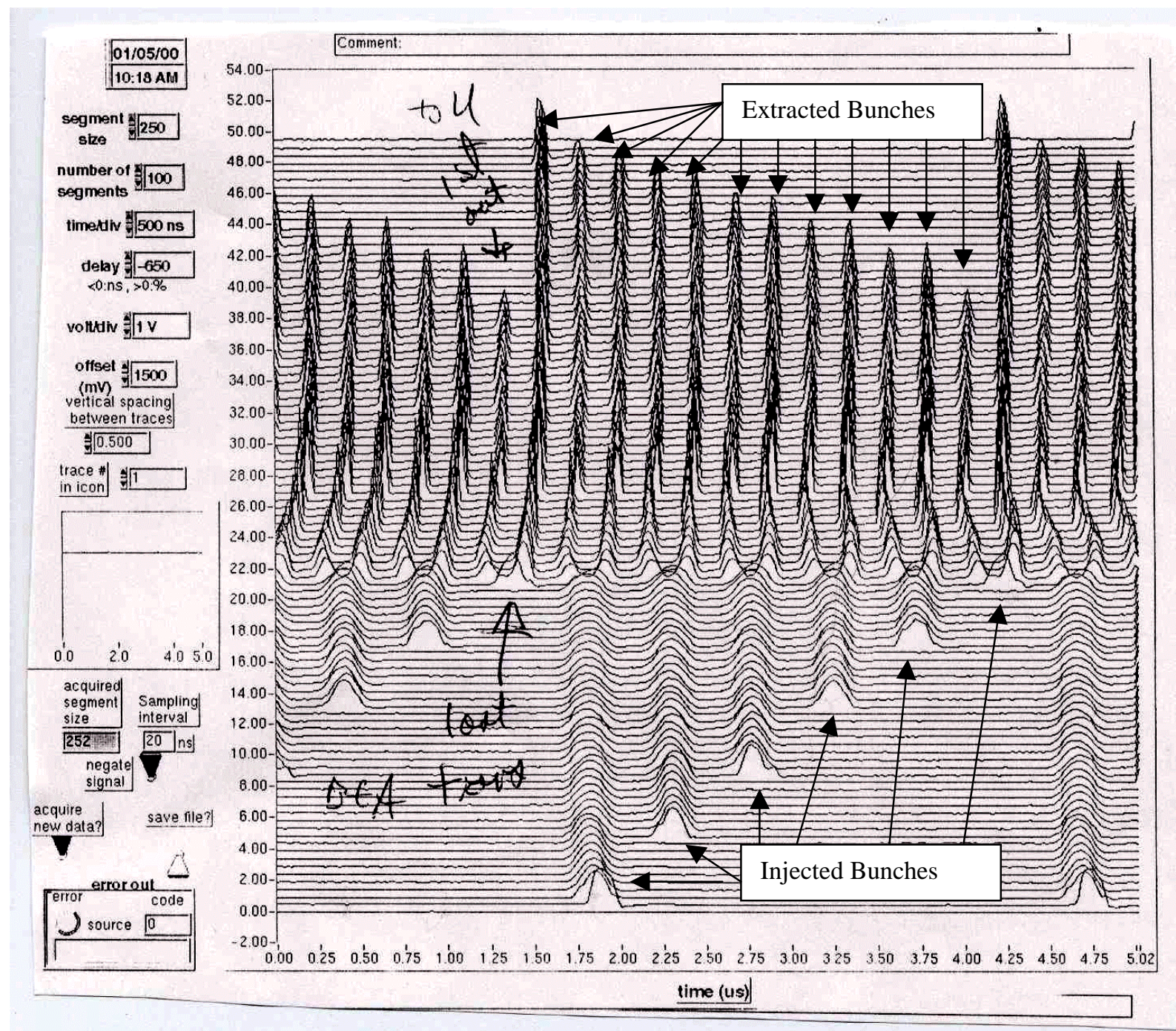

Figure 1: "Mountain Range" plot of the longitudinal charge dencity, as is measured by the "Wall Current Monitor", of each bunch in the AGS as a function of time during an AGS cycle. Each horizontal trace corresponds to two revolutions around the AGS ring ( $\sim \mu \mathrm{sec})$. The time interval between horizontal traces (vertical axis) is $\sim 15 \mathrm{msec}$. The bottom of the plot corresponds to the injection time, and shows the sequential injection of the six bunches from the AGS-Booster into the AGS. Note the injection time interval is $\sim 150 \mathrm{msec}$.

The bunch splitting from six into twelve begins at $\sim 800 \mathrm{msec}$ from the injection of the first bunch.

Following the acceleration each of the twelve bunches is extracted from the AGS sequentially (note the missing mountain in the vertical direction on the plot). 


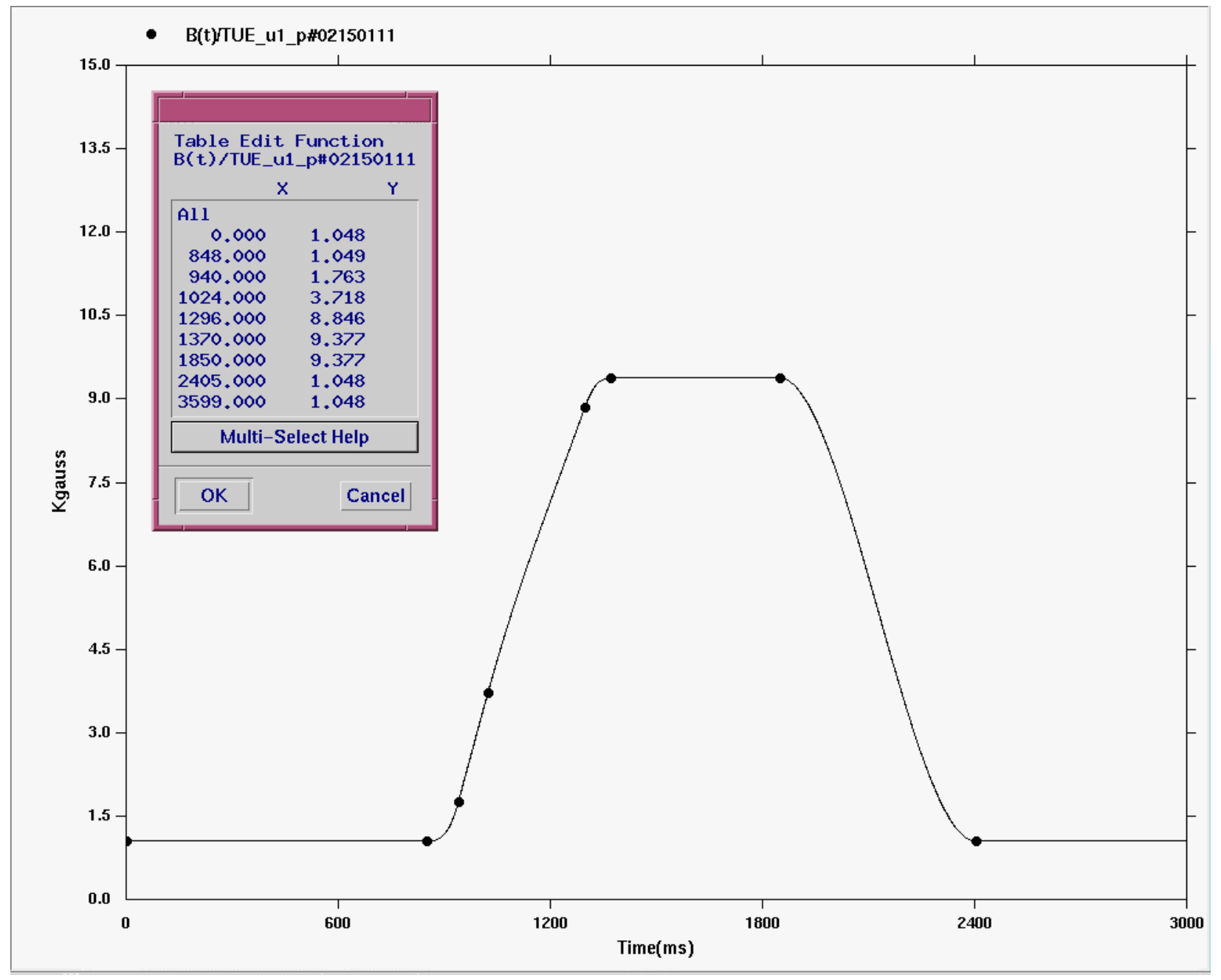

Figure 2: $\quad$ Main-Magnet cycle of the AGS syncrotron. Beam Injection from the Booster and subsequent bunch splitting (see text) occurs in the "Front Porch" from 0 to $850 \mathrm{msec}$. Fast Beam Extraction from the AGS takes place in the "Flat top" from $1370 \mathrm{msec}$ to $1850 \mathrm{msec}$ 

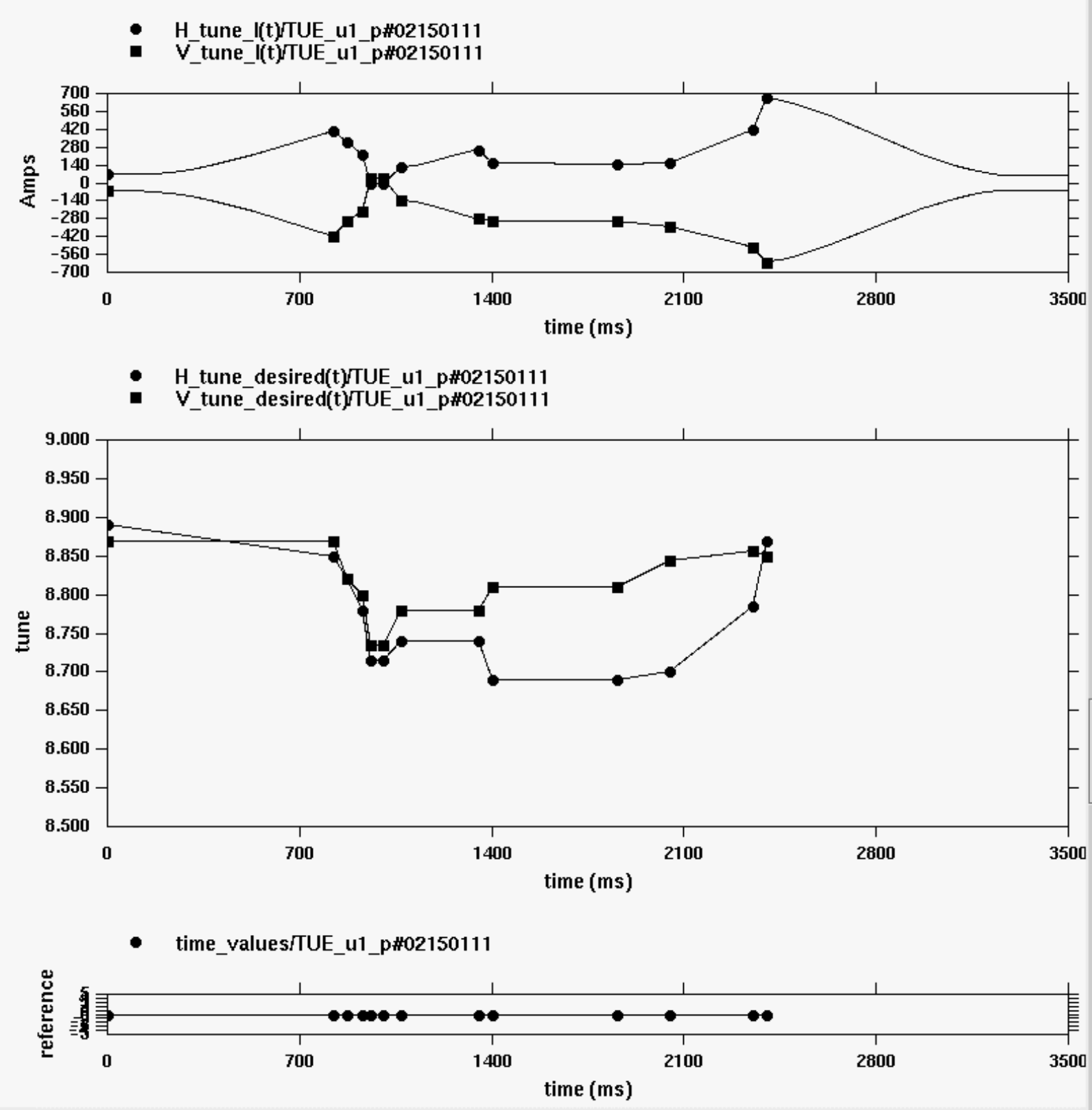

Figure 3: Horizontal and vertical tunes of the AGS synchrotron during the magnet cycle (middle graph). The currents of the horizontal and vertical quadrupoles that control the tunes (tune quadrupoles) are also plotted (top graph). 


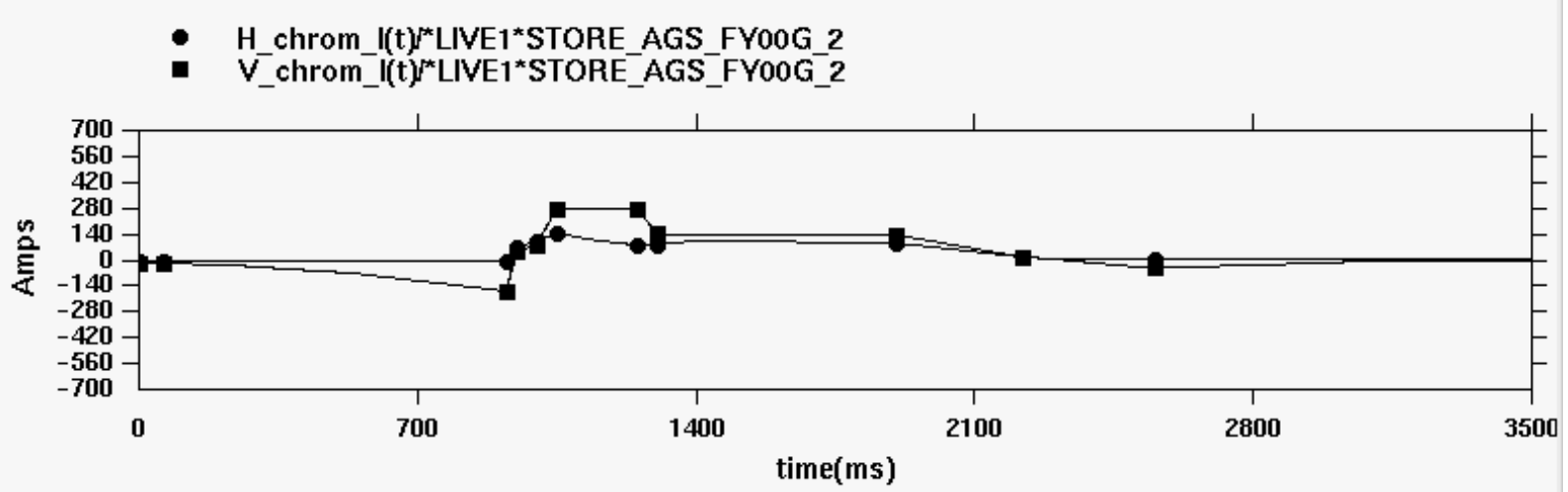

- H_chrom_desired(t) r $^{*}$ LIVE1*STORE_AGS_FY00G_2

- V_chrom_desired(t) VIVIVE1 $^{\star}$ STORE_AGS_FYOOG_2

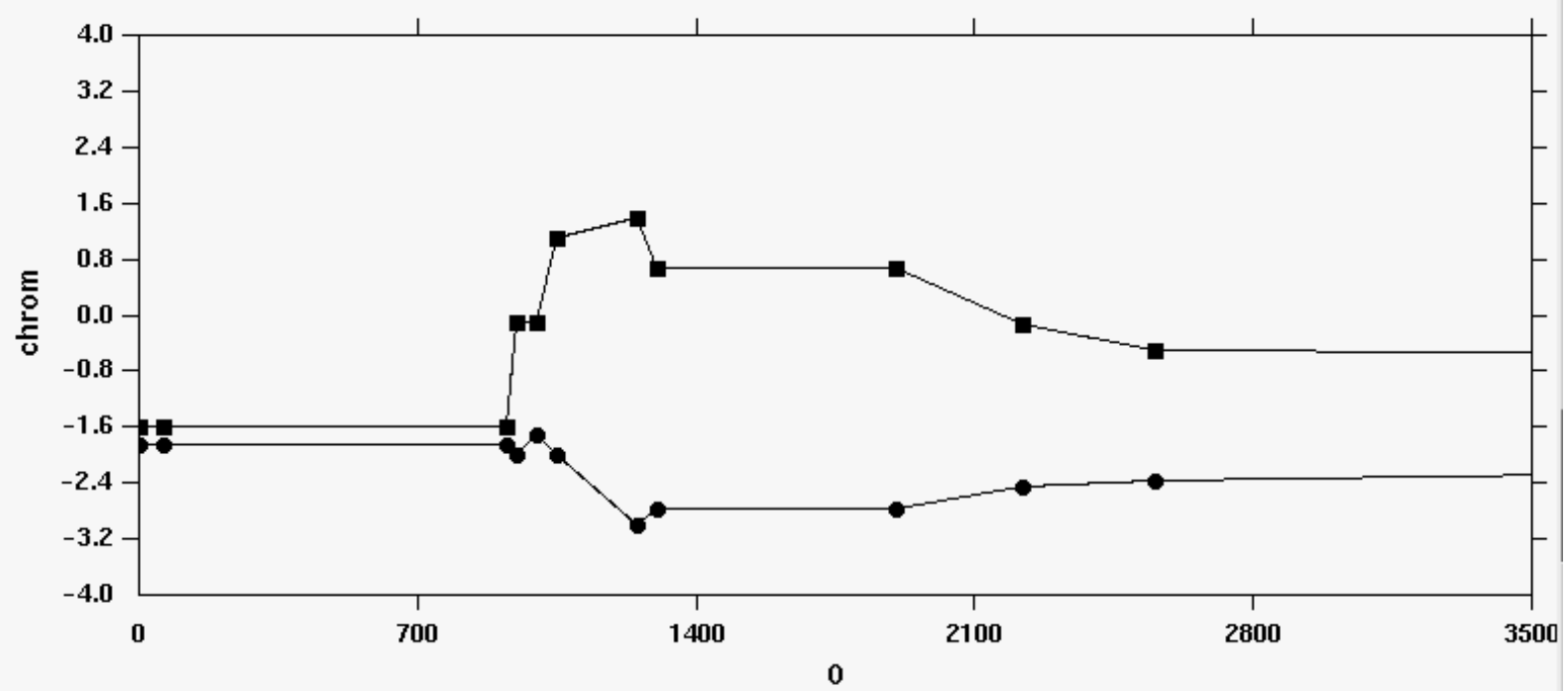

- time_values/*LIVE1*STORE_AGS_FYO0G_2

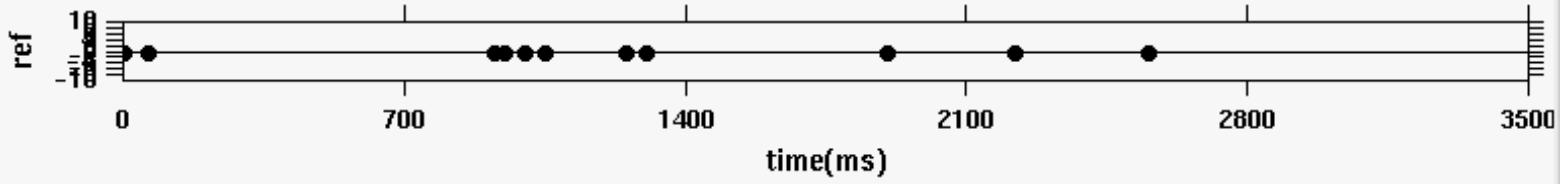

Figure 4: Horizontal and vertical chromaticities of the AGS synchrotron during the magnet cycle (middle graph). The currents of the horizontal and vertical sextupoles that control the chromaticities (chromaticitiy sextupoles) are also plotted (top graph). 


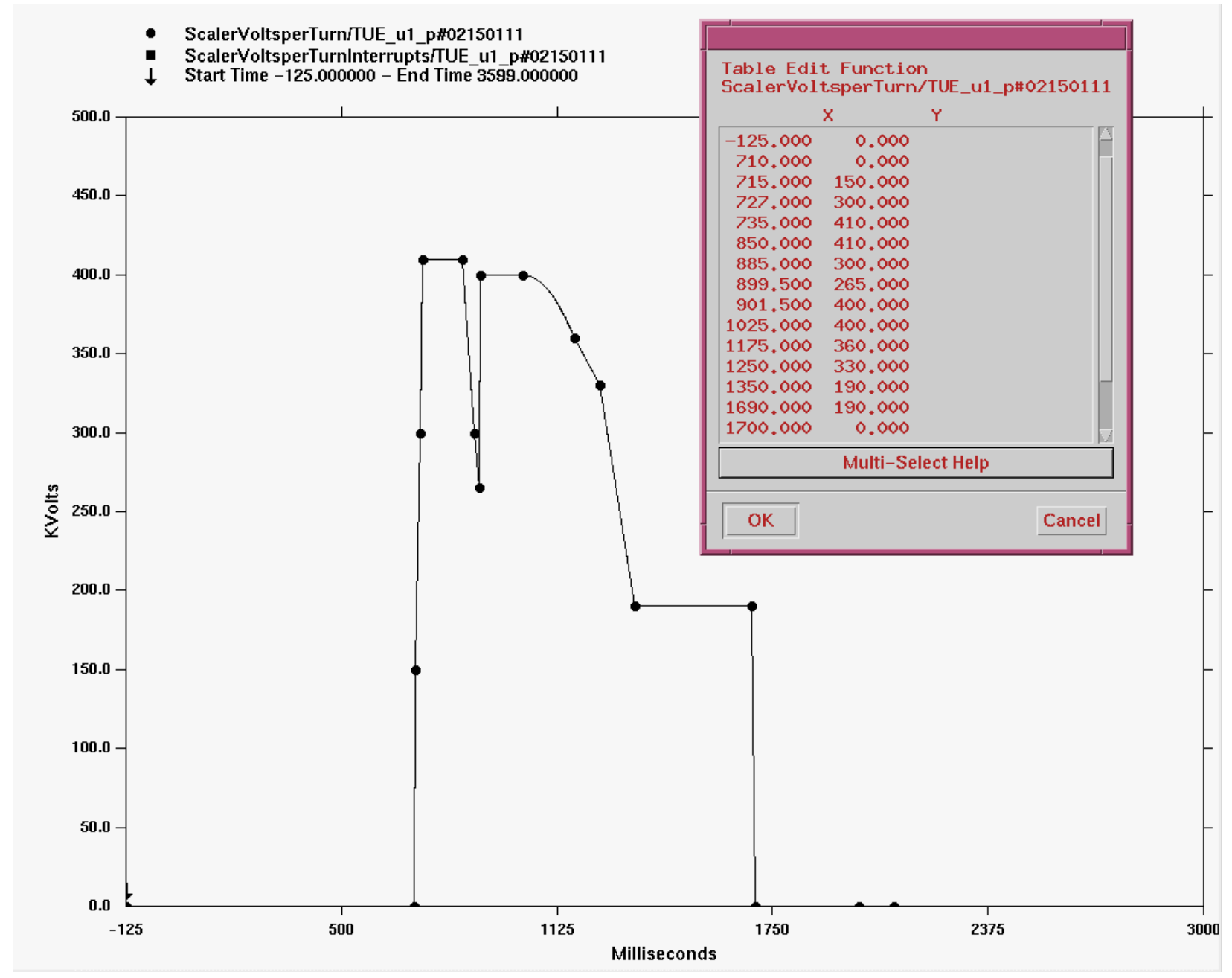

Figure 5: The Gap-Volts of the $\mathrm{h}=12$ AGS RF Cavities as a function of time during the AGS Magnet cycle 


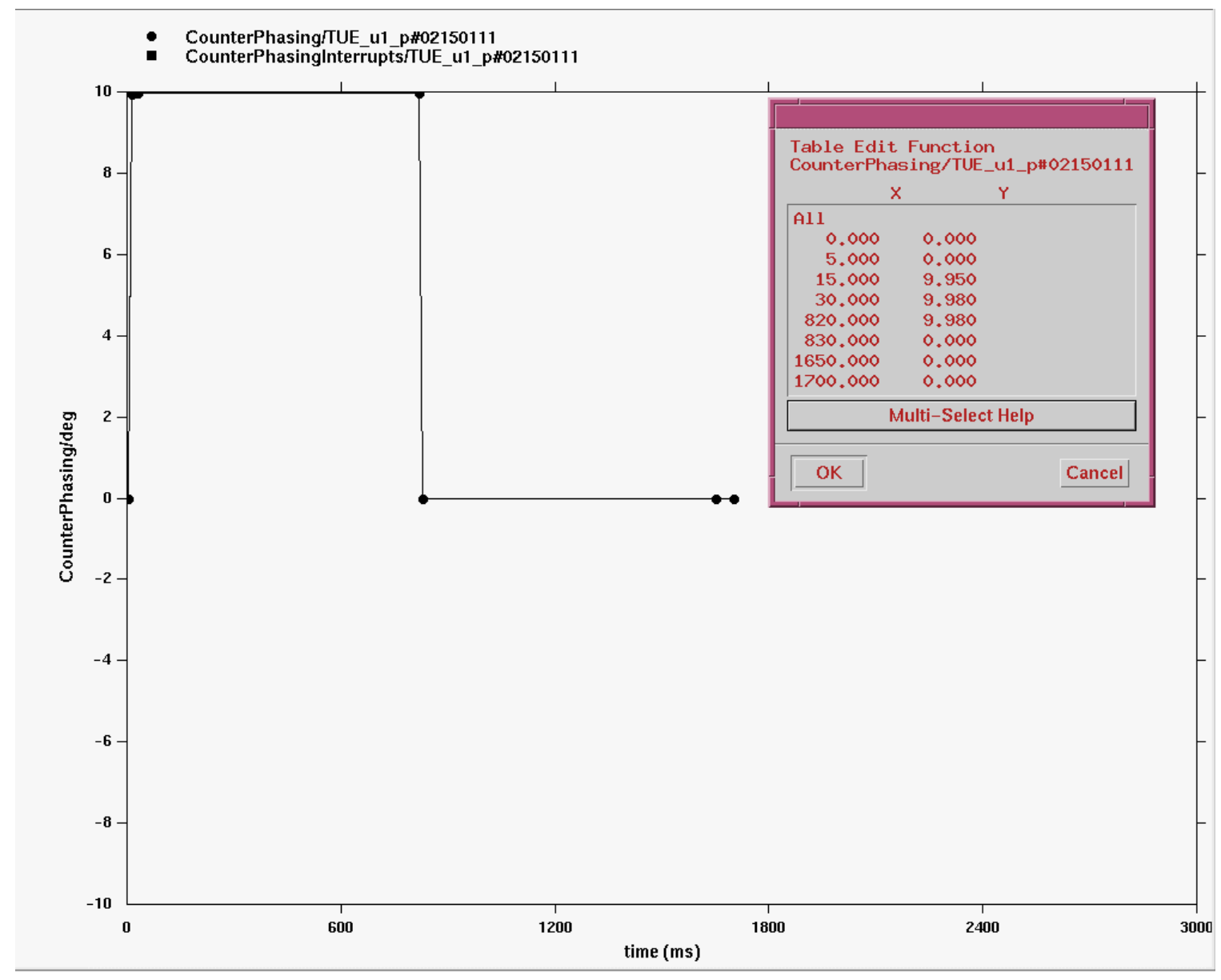

Figure 6: Counterphasing of the $\mathrm{h}=6$ AGS RF Cavities as a function of time during the AGS main-magnet-cycle. 


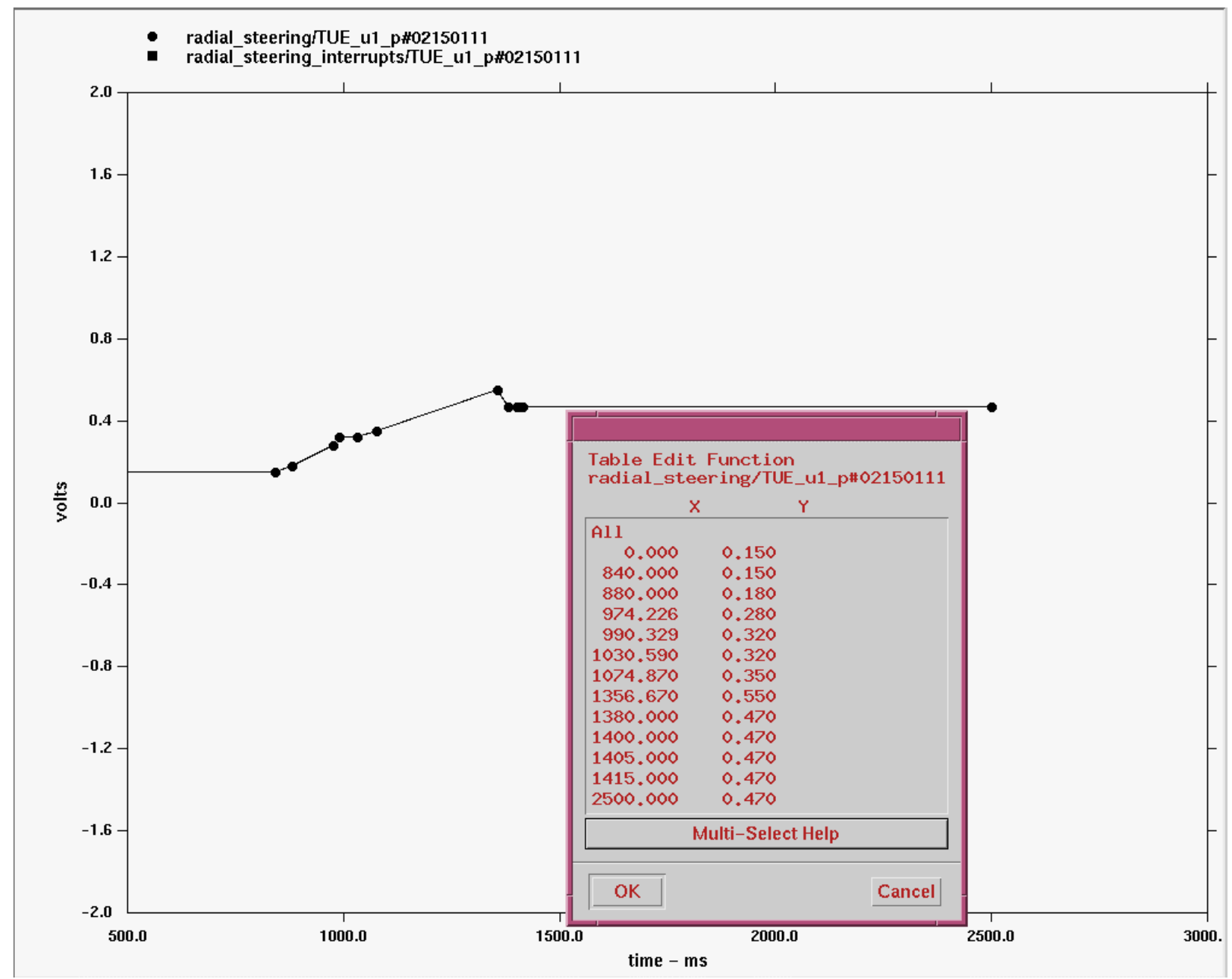

Figure 7: The radial steering of the beam as a function of time during the AGS mainmagnet-cycle. 


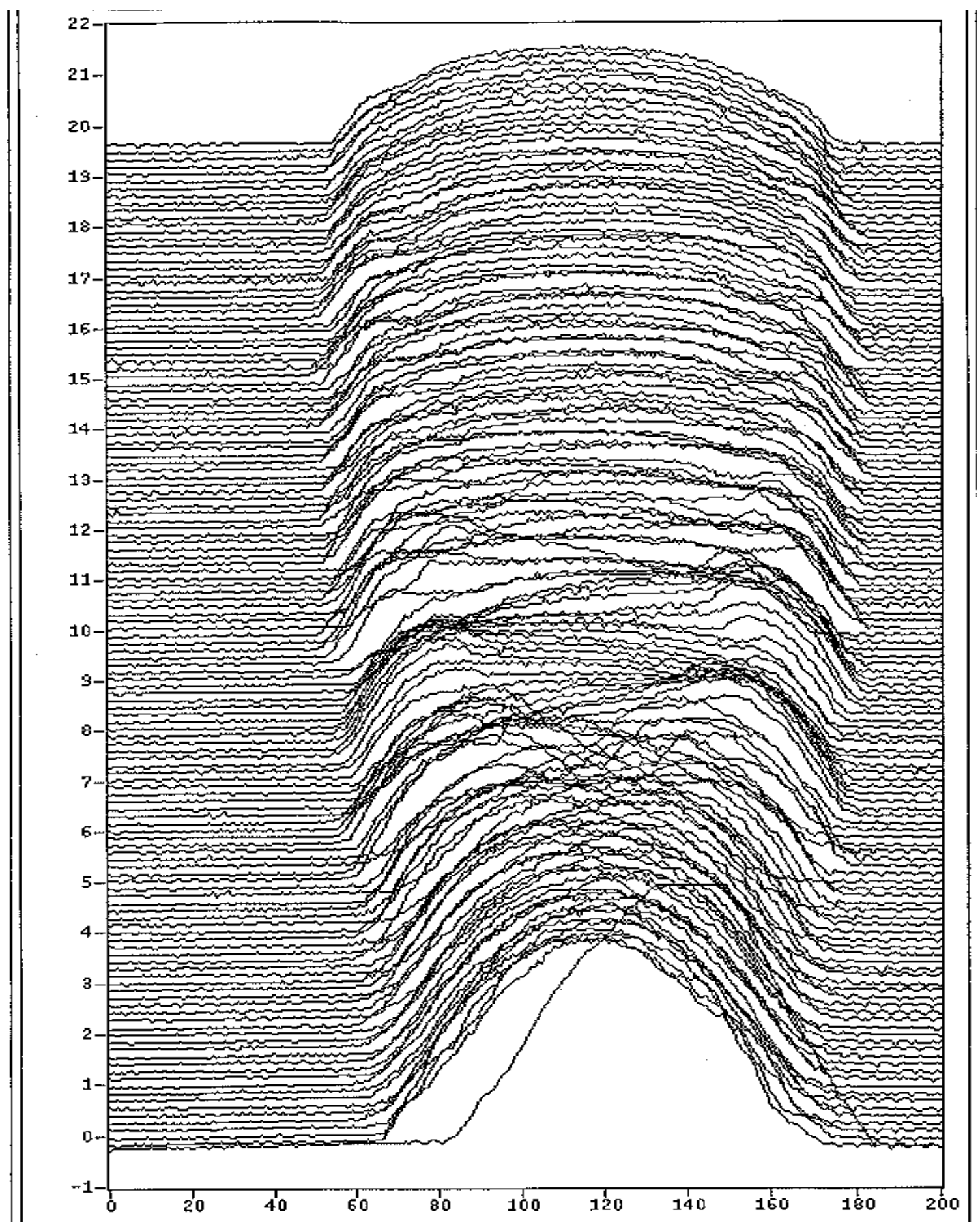

Figure 8: $\quad$ Isometric plot of the charge distribution of a beam bunch, as measured by wall current monitor, when the bunch passes by (horizontal-axis). Each trace in the plot corresponds to a bunch revolution. The width of the bunch is $\sim 100 \mathrm{nsec}$ as shown by the horizontal scale. The effect of the VHF cavity is apparent by the smoothness of the charge distribution which is shown higher up in the graph. 


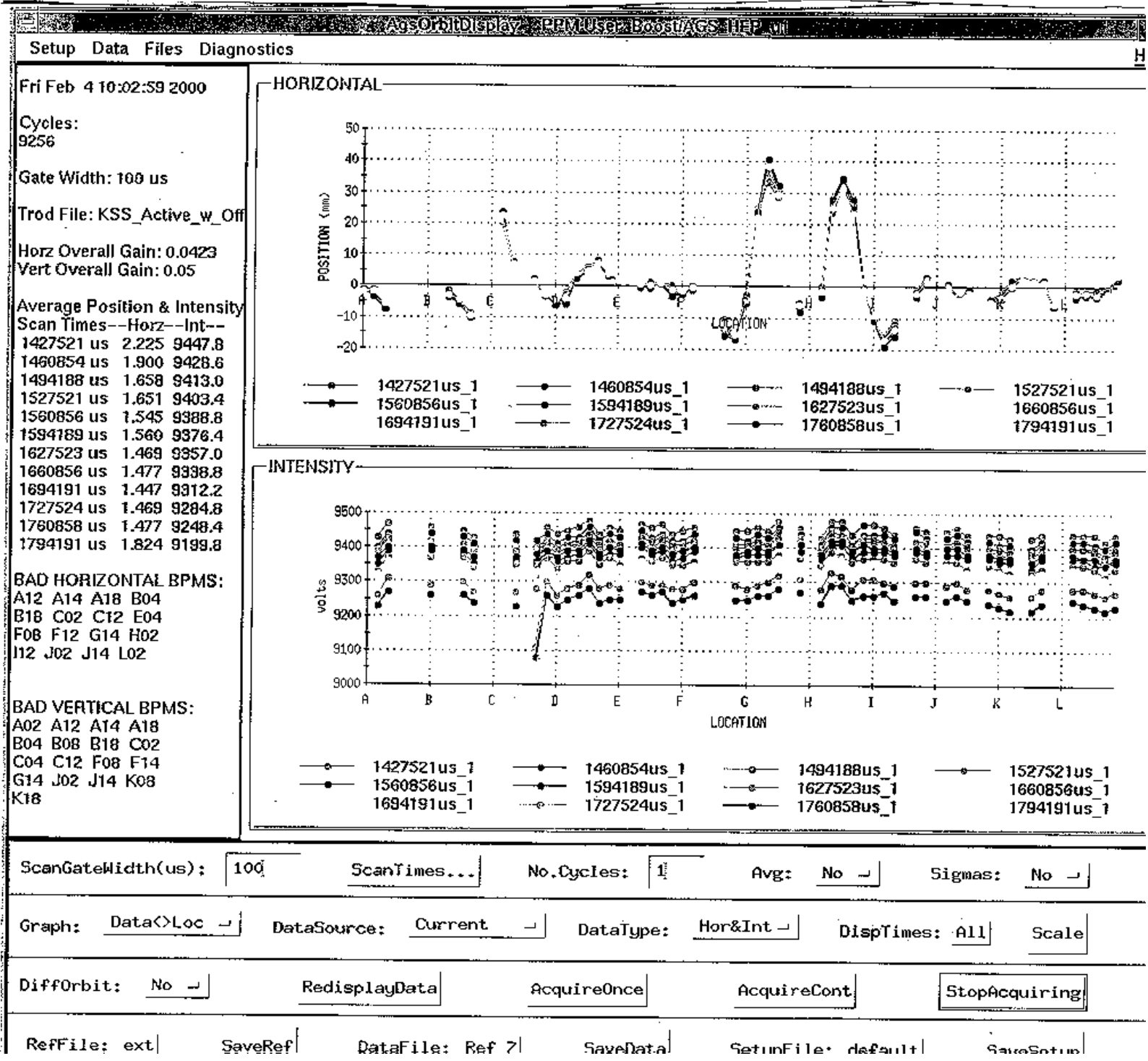

Figure 9: $\quad$ Beam orbits in the AGS (top-graph) during fast beam extraction. There are 12 orbits which were taken at times (as measured from $\mathrm{T}_{0}$ ) shown in the bottom of the graph. These times coincide with the maximum beam displacement at the $\mathrm{G}$ and $\mathrm{H}$ straight sections of the AGS. The G09 and H11 "orbit bumps" (see text) are clearly shown. Displacement "zero" corresponds to a beam orbit coinciding with an orbit at $\mathrm{R}_{0}$. Positive beam displacements correspond to orbits with radii $\mathrm{R}>\mathrm{R}_{0}$.

The bottom graph shows the total beam current of the beam bunches circulating in the AGS as function of the BPM location. The beam current is measured by the BPM's. Note that the currents measured at different times, is reduced by an amount corresponding to the current of the previously extracted bunch. 


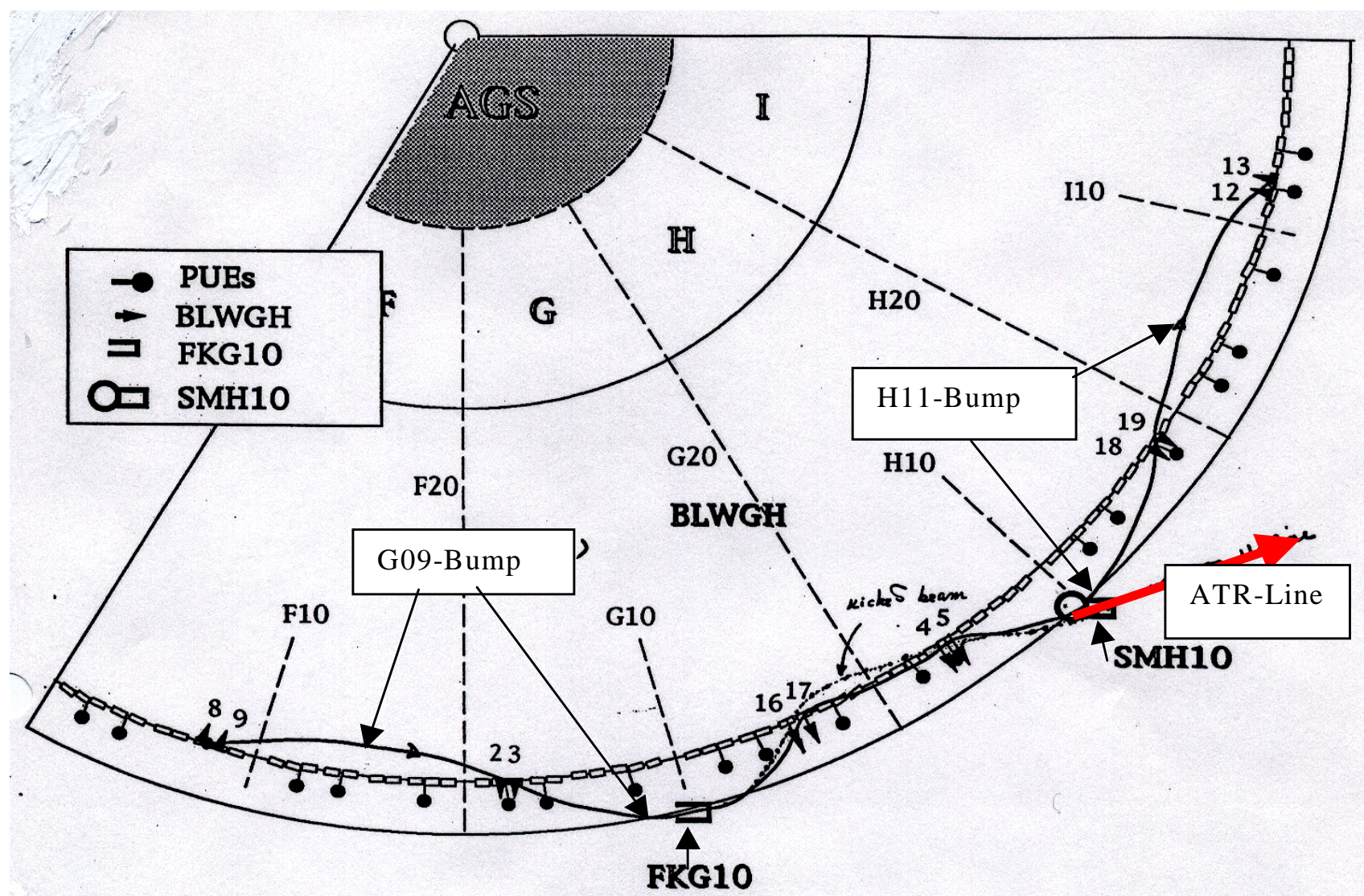

Figure 10: Schematic diagram of the AGS's FEB section showing the relative location of the extraction devices the "bumped orbit" and the extraction trajectory. 


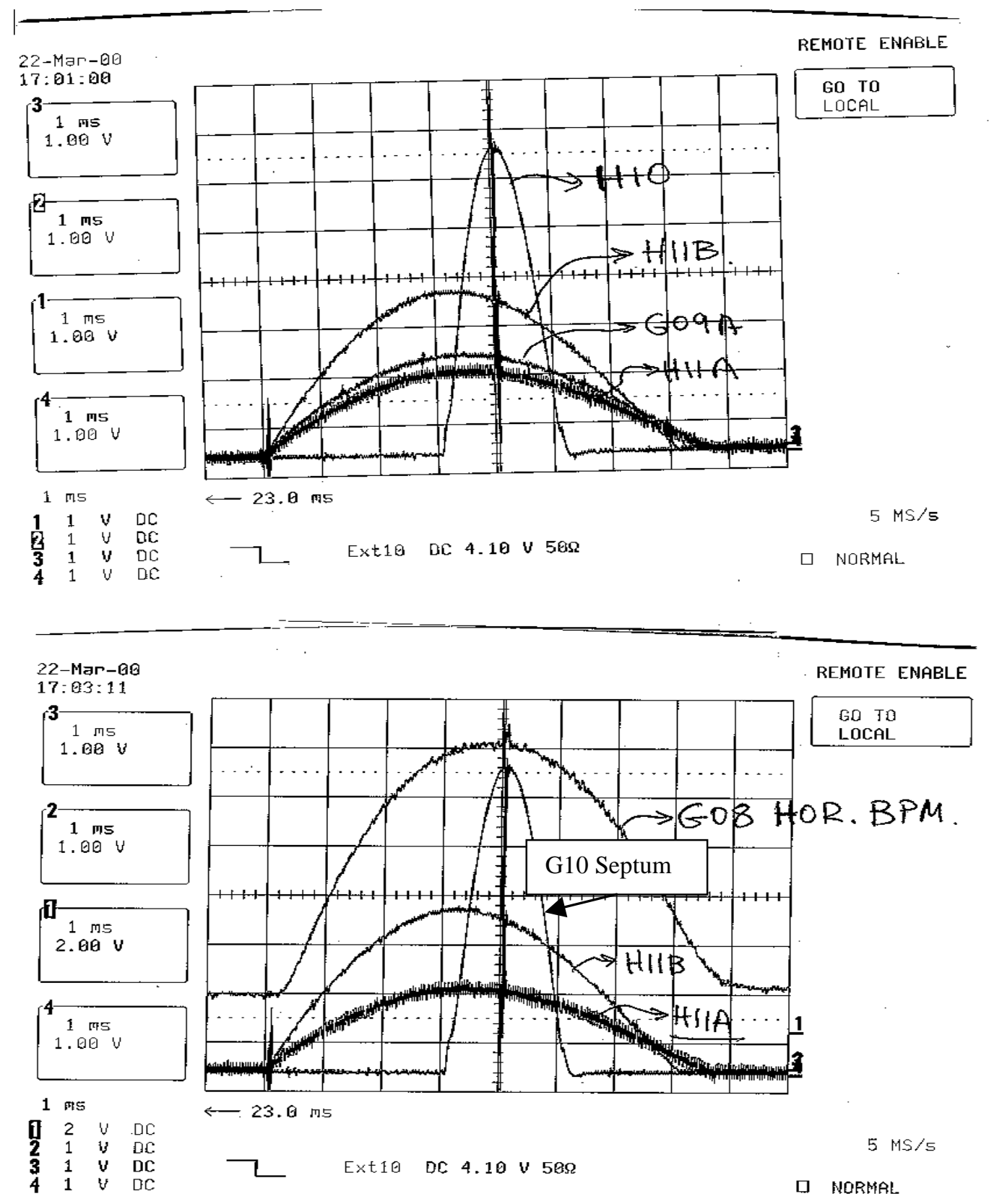

Figure 11: Oscilloscope traces (top) of the current of the "back-leg-windings" of the G09_bump (G09A) and the H11_bump (H11A, H11B) and the current of the H10 septum. The oscilloscope traces of the bottom graph show the horizontal displacement of the beam as measured by the G08_BPM. Note that the maximum beam displacement as measured by the G08_BPM, occurs at a later time $(\sim 0.5 \mathrm{msec})$ than the maximum current in the "back-leg-windings". The firing time of the $\mathrm{H} 10$ septum is adjusted to provide maximum beam displacement at the first BPM of the U-line. The vertical line (top and bottom) which runs through the maximum of current pulse of the H10 magnet, coincides with the time that the G10 kicker fires. 


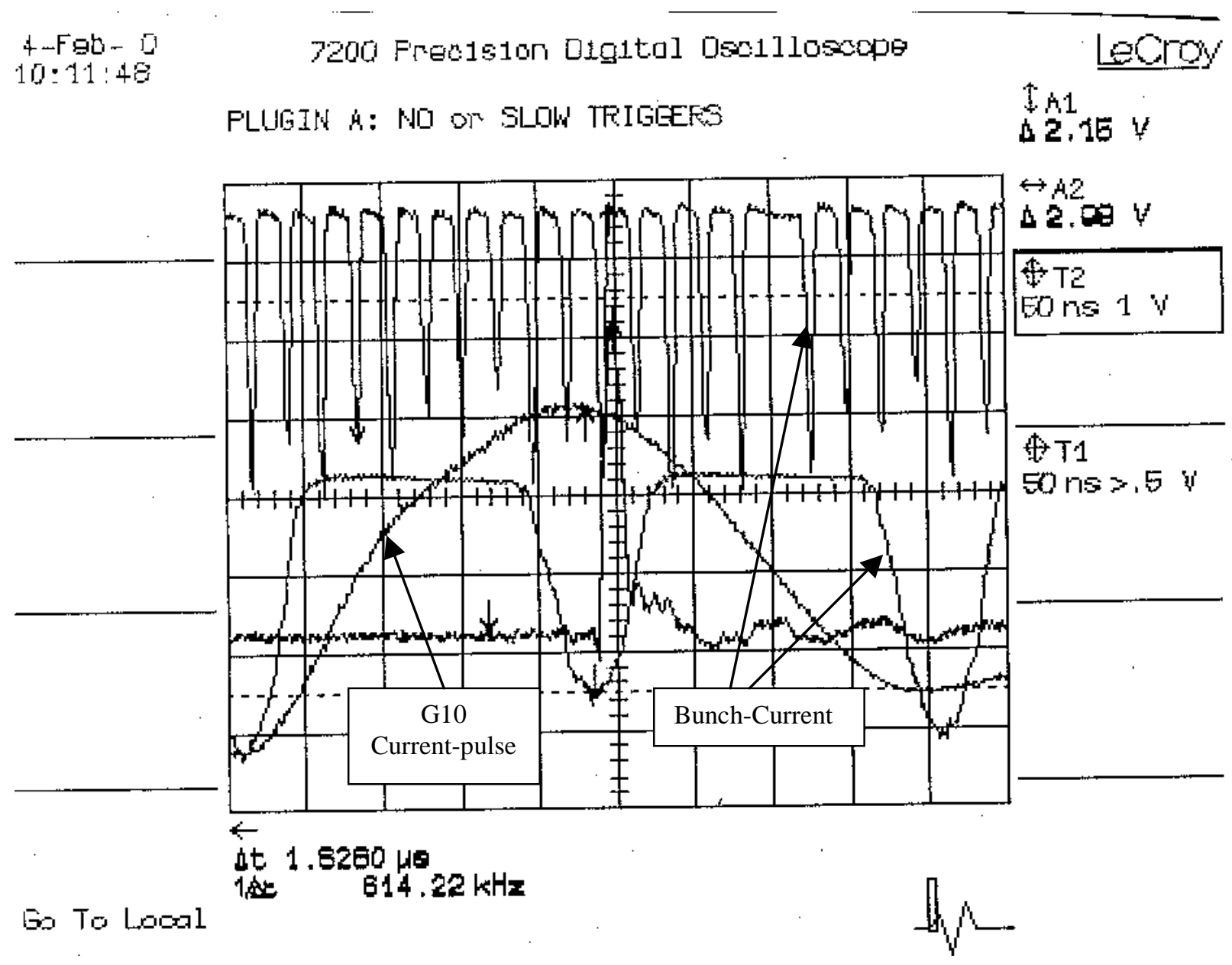

Figure 12: An oscilloscope trace of the circulating beam bunches is shown by the "negative spikes" displayed on the top of the graph and correspond to the longitudinal charge distribution of the beam bunches as measured by the "wall current monitor". A time-expanded view (50 nsec/div) of three consecutive beam bunches is also shown in the same graph. The positive "half-sine-wave" curve in the graph corresponds to the kicker current which is displayed in 50 nsec/div scale. 
HF 5371A Frequency and Time Interval Analgzer

Graph shous 440 meas ( $88 \%$.

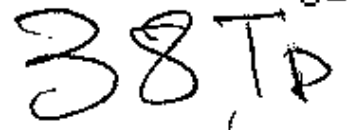

Hist: Time Int A $\rightarrow \mathrm{B}$

- Mkr $x:$ G2. 0717 us

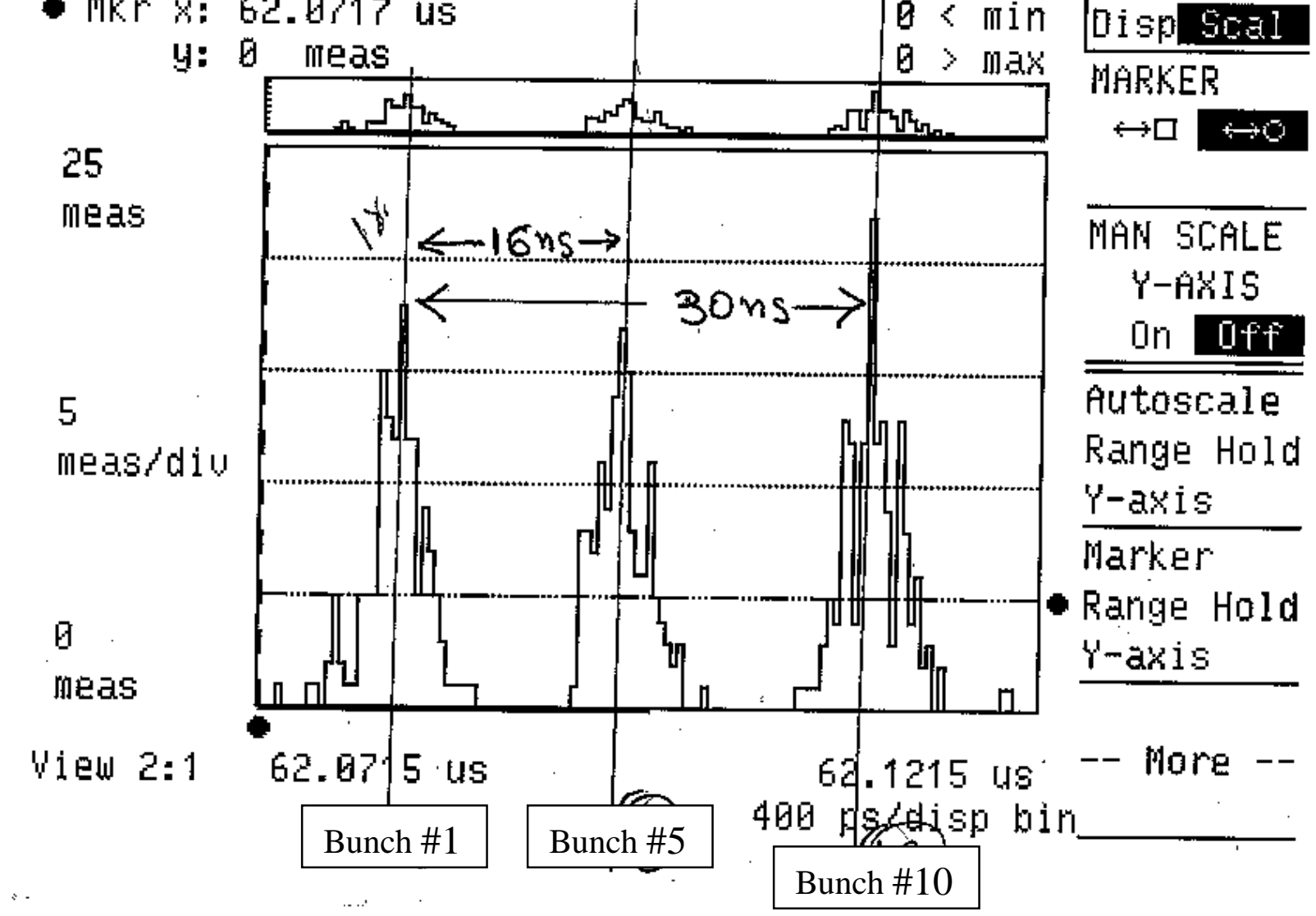

Figure 13: Time distribution of the "time-defference" between the ARF.FEBREQ.DELAY trigger, and the "G10 kicker firing" trigger, for the extraction of the first bunch (first peak) the fifth bunch (second peak) and the tenth bunch (third peak).

The integrated current of all 12 bunches circulating in the AGS was $38 \times 10^{12}$ protons. 


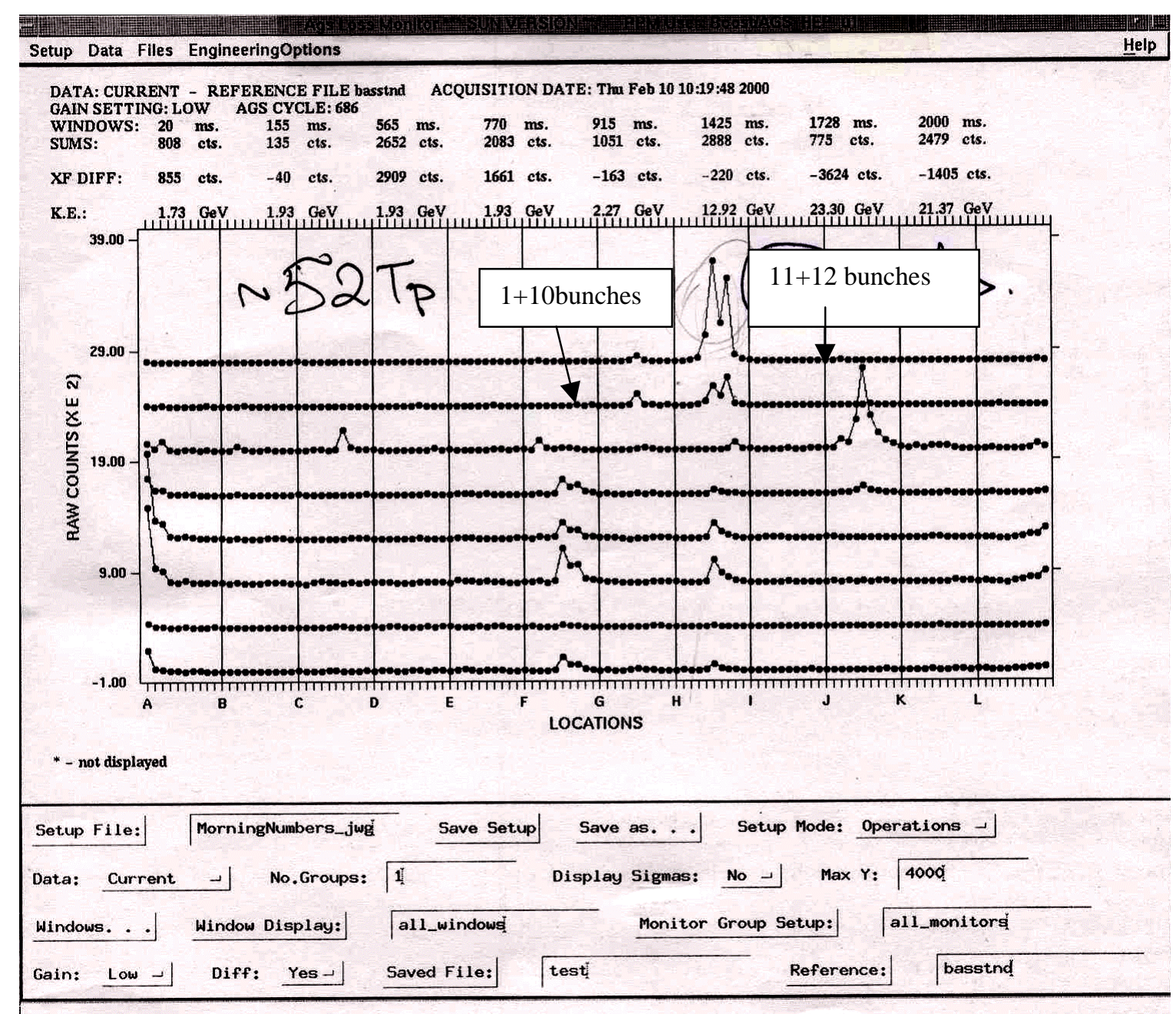

Figure 14: Radiation levels at various time intervals of the AGS cycle, as a function of the azimouthal location in AGS. The curve labeled " $1+10$ bunches" corresponds to the radiation levels along the ring (horizontal axis), in the interval $1425 \mathrm{msec}$ to $1728 \mathrm{msec}$ from $\mathrm{T}_{0}$. In this time interval the first 10 bunches are extracted from the AGS. The radiation levels during the extraction of the last two bunches are shown by the curve " $11+12$ bunches". The radiation levels of the " $11+12$ " are higher then the levels of the " $1+10$ " because the timing of the last two bunches cannot be synchronized with the firing time of the G10 kicker. 

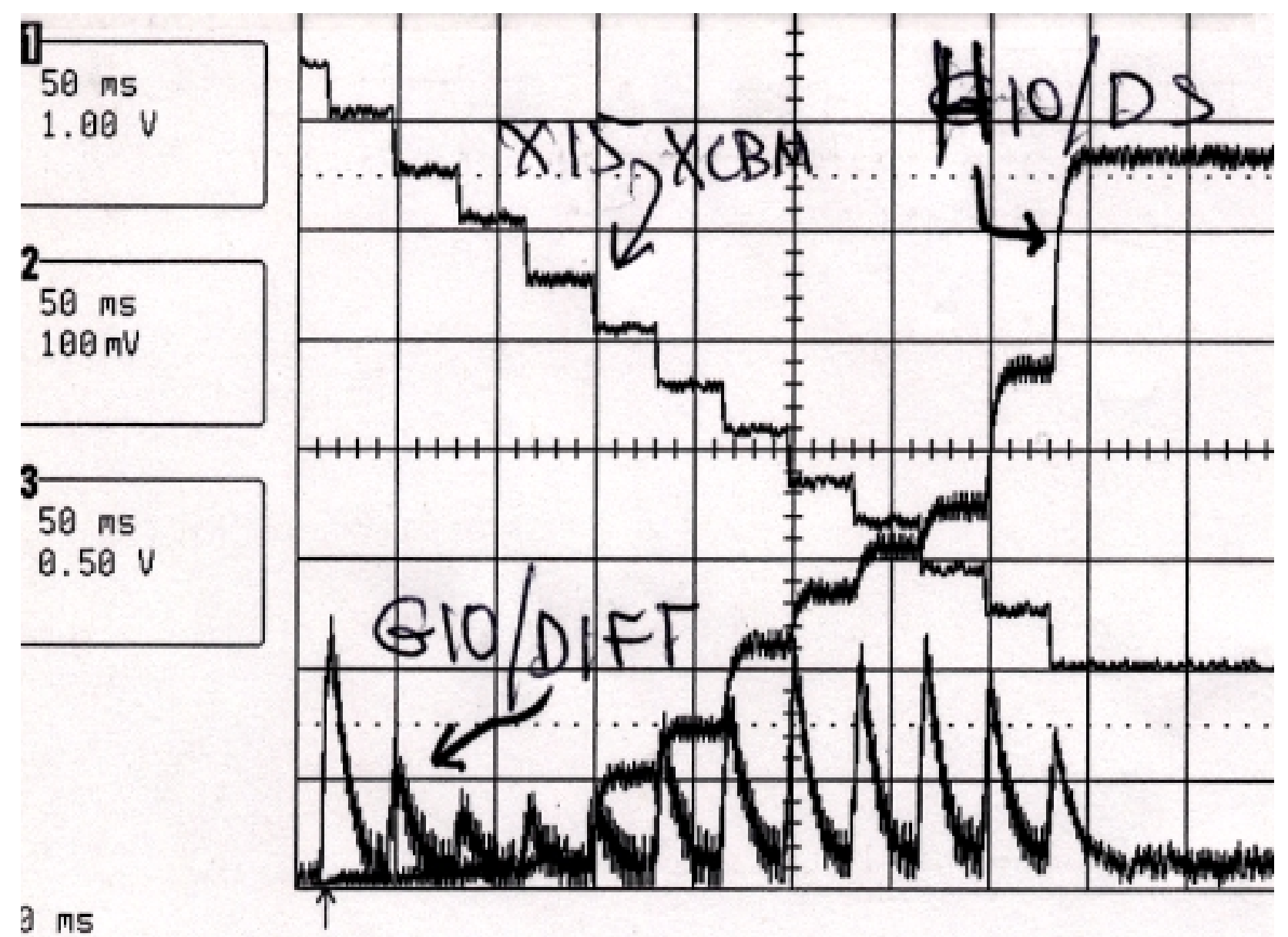

\section{a m5}

$1 \cup \quad D C$

$.1 \quad V \quad D C$

$.5 \cup \quad D C$

$.5 \cup \quad D C$

\section{Ext10 OC $2.50 \cup 50 \Omega$}

Figure 15: Radiation levels, during the extraction of the 12 bunches from the AGS, recorded by the H10/DS and G10/DF. The descending staircase represents the beam current circulating in the AGS and monitored by the X15-XCBM current transformer. 


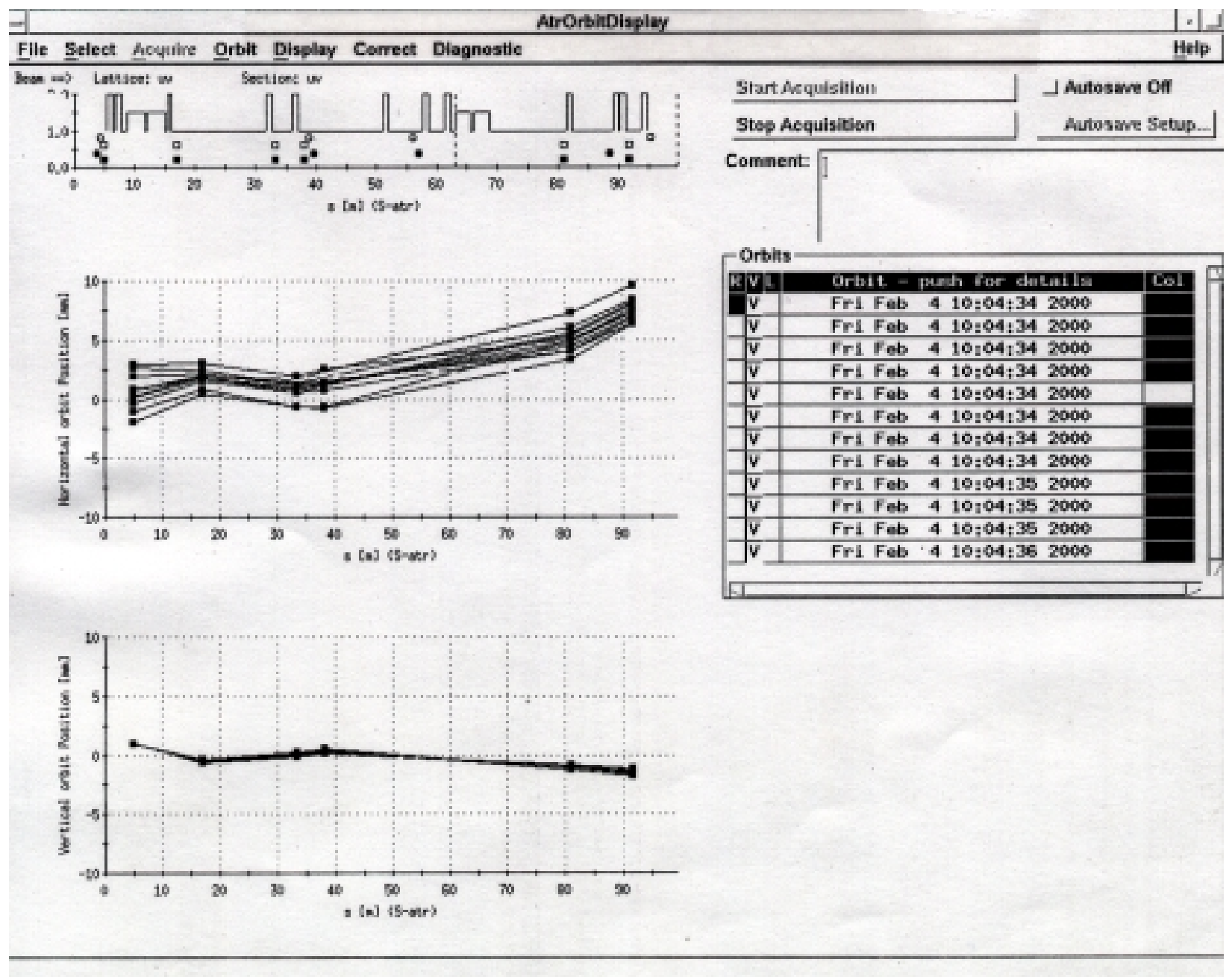

Figure 16: The horizontal (curves on top graph) and vertical (bottom graph) "beam trajectories" of each of the 12 extracted bunches at specified locations along the U-V transfer line. The specified locations are defined by the BPM's of the U-V line, which measure the horizontal and vertical location of center-of-charge of each bunch at the center of the BPM's. The solid lines are not the actual trajectories but are drawn to guide the eye 


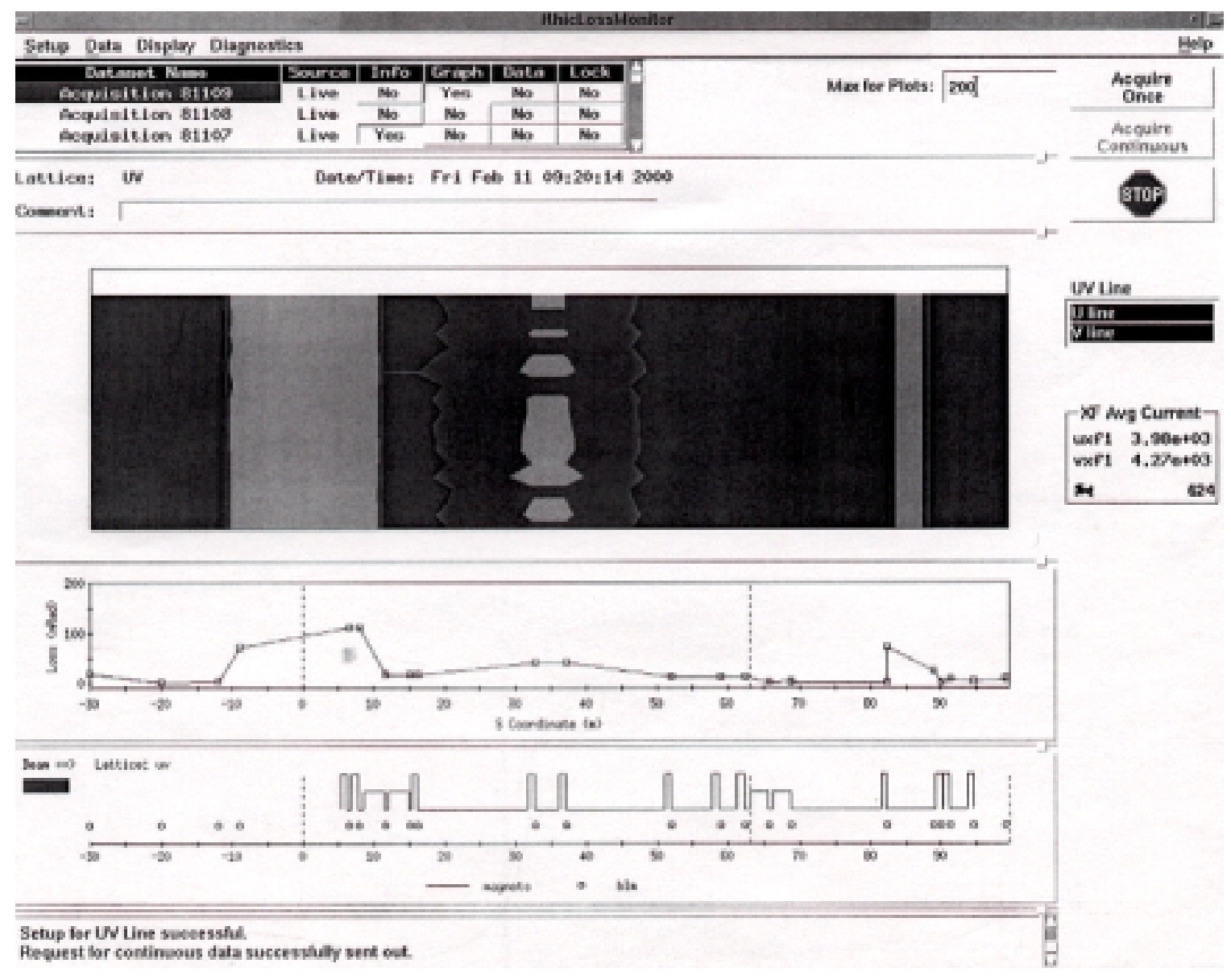

Figure 17: Radiation levels (vertical axis) along the U-V-line during the transport of the beam bunches from the AGS to the V-target. The radiation levels at the location " $0 \mathrm{~m}$ " of the horizontal axis are due to the beam extraction (see fig.12). The radiation levels at the location "80 m" are due to a "vacuum window" which is placed at this location. 


\section{$\mathrm{X}, \mathrm{Y}$ beam profile UV-LINE}

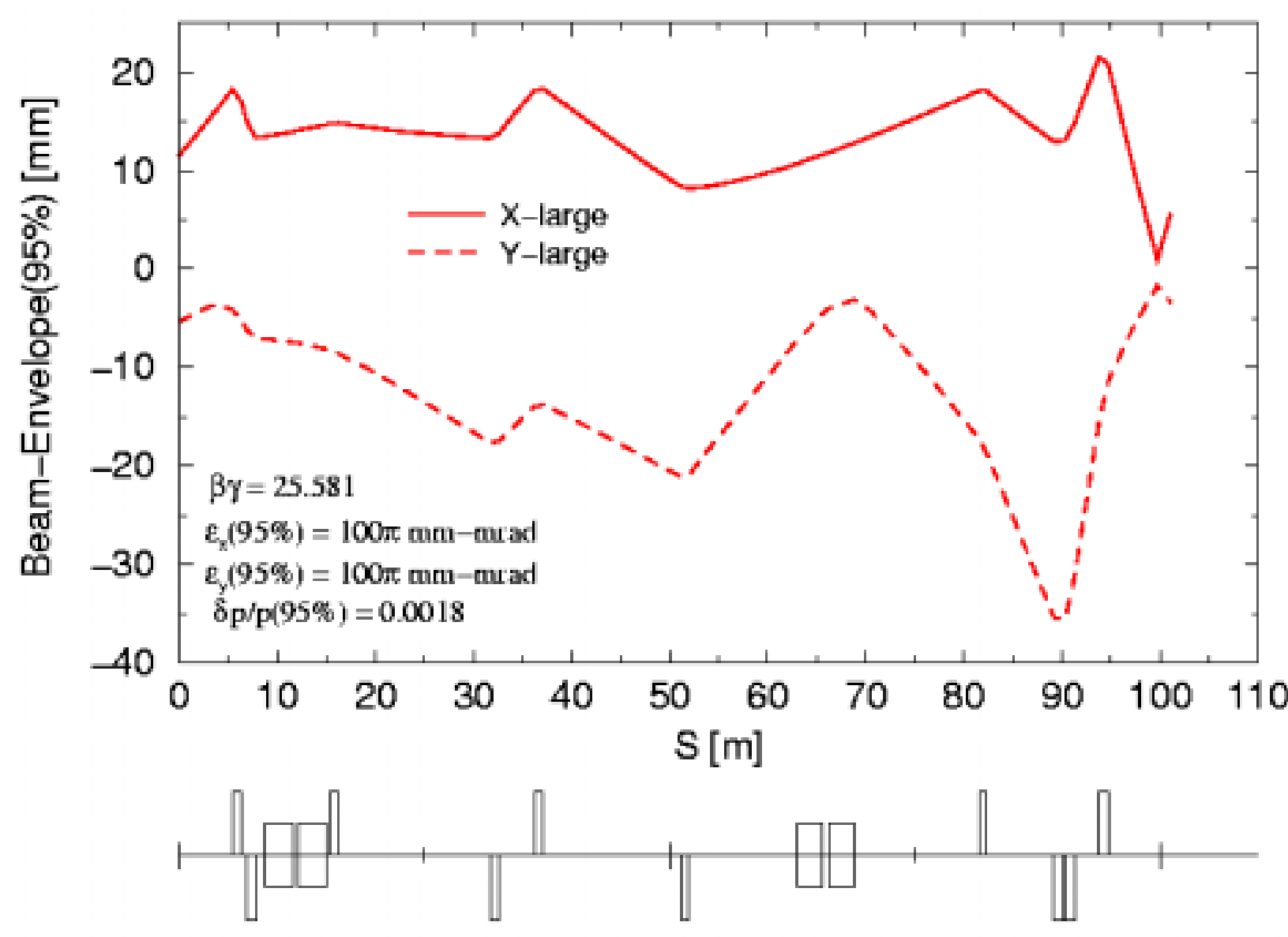

Figure 18: The horizontal $(\mathrm{X})$ and vertical $(\mathrm{Y})$ beam profiles of the beam in the U-V-line. The beam profiles correspond to a $95 \%$ normalized beam emittance of $\varepsilon_{\mathrm{x}, \mathrm{y}}=100$ pi.mm.mrad 


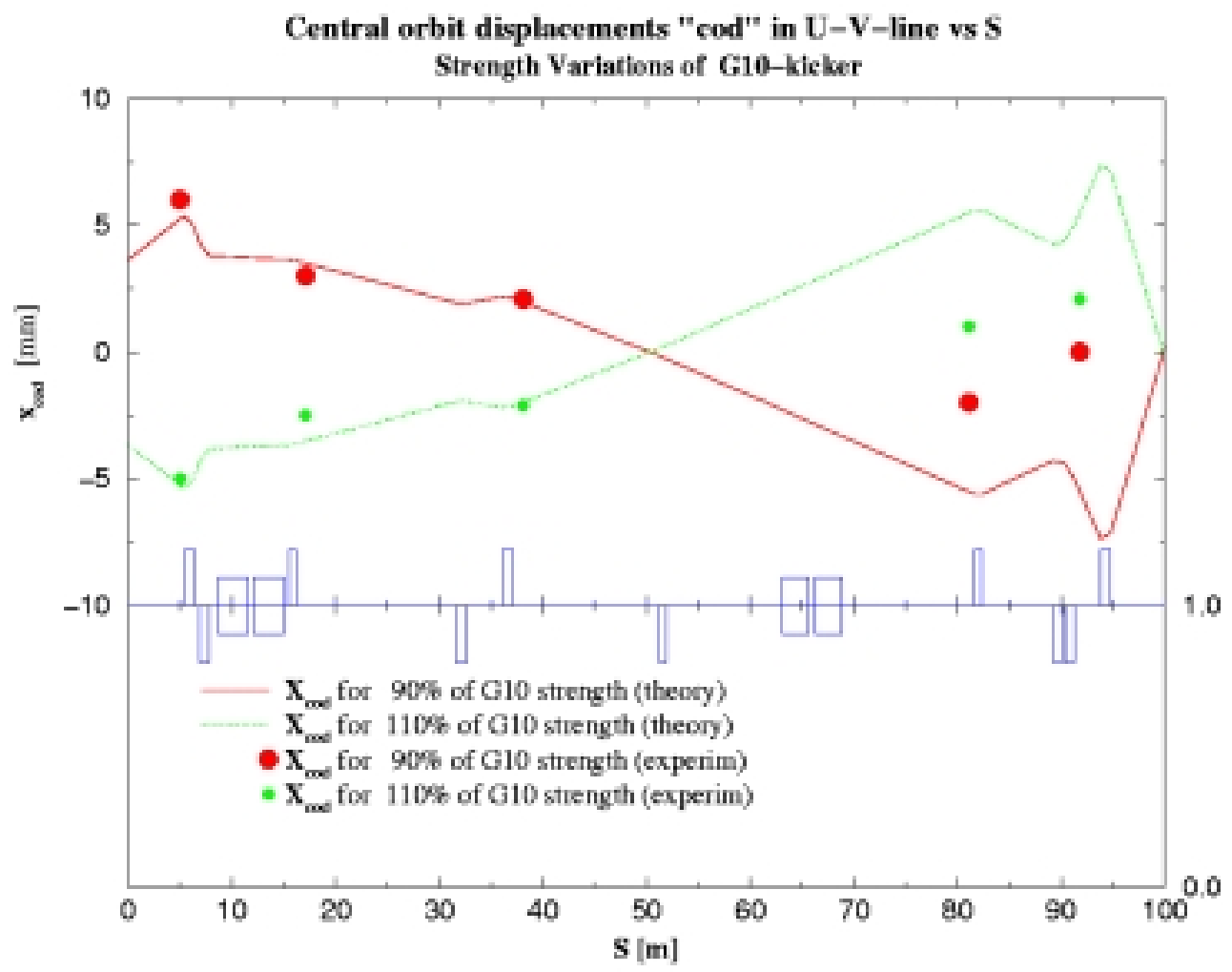

Figure 19: Horizontal (X) Central orbit displacements $X_{\text {cod }}$ along the $U-V$ line. The $X_{\text {cod }}$ is due to variations of G10 strength. The solid and dotted lines are theoretical predictions. The solid dots are experimental values of $\mathrm{X}_{\mathrm{cod}}$ obtained from BPM readings. 


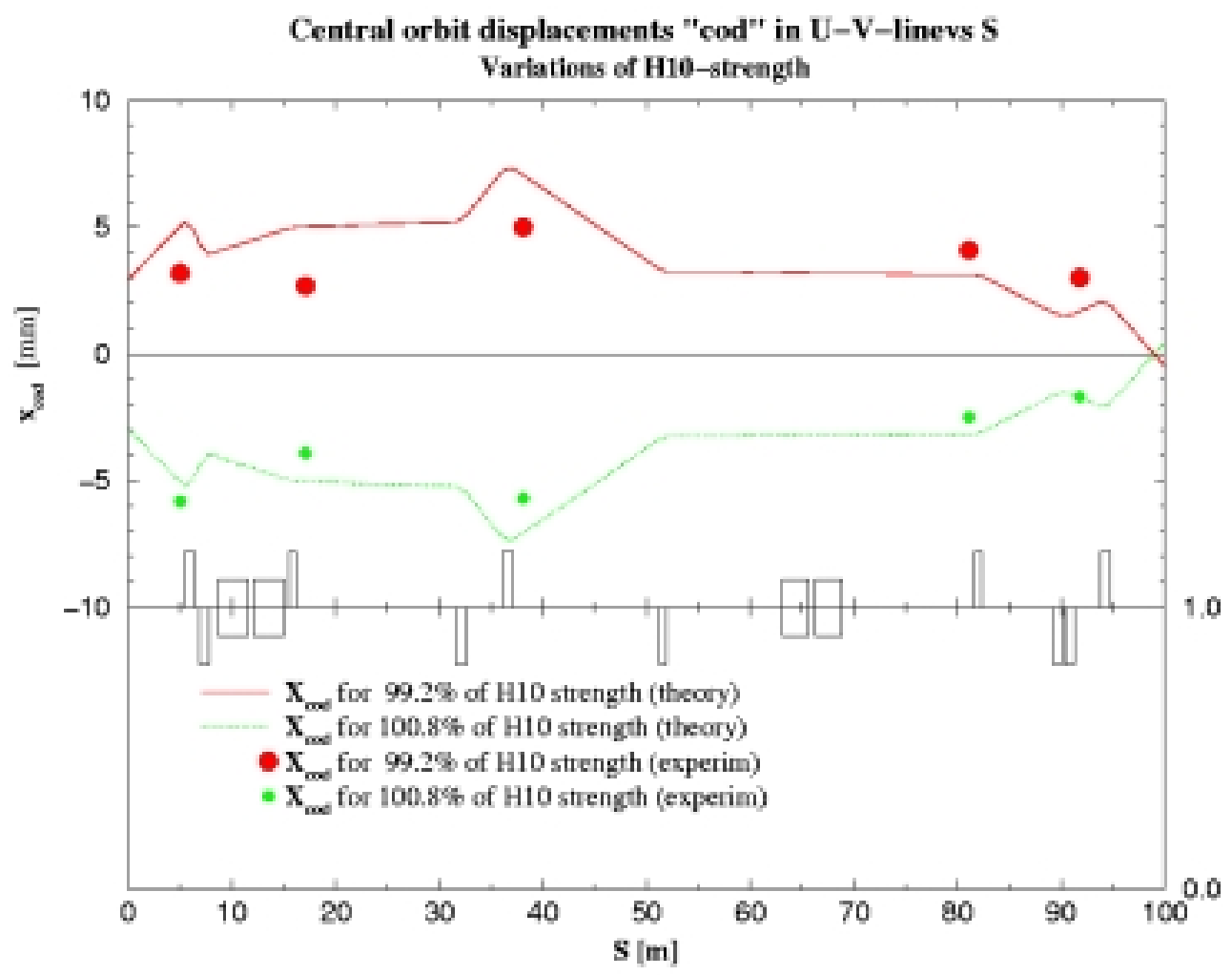

Figure 20. Horizontal (X) Central orbit displacements $\mathrm{X}_{\text {cod }}$ along the $\mathrm{U}-\mathrm{V}$ line. The $\mathrm{X}_{\mathrm{cod}}$ is due to variations of G10 strength. The solid and dotted lines are theoretical predictions. The solid dots are experimental values of $\mathrm{X}_{\mathrm{cod}}$ obtained from BPM readings. 